\title{
A latent variable approach to potential outcomes for emergency department admission decisions
}

\author{
Amy L. Cochran ${ }^{1}$ | Paul J. Rathouz ${ }^{2}$ | Keith E. Kocher ${ }^{3}$ | Gabriel Zayas-Cabán미
}

${ }^{1}$ Department of Biostatistics and Medical Informatics, University of

Wisconsin-Madison, Madison, Wisconsin

${ }^{2}$ Department of Population Health,

The University of Texas at Austin, Austin,

Texas

${ }^{3}$ Department of Emergency Medicine, University of Michigan Medical School, Ann Arbor, Michigan

${ }^{4}$ Department of Industrial and Systems Engineering, University of

Wisconsin-Madison, Madison, Wisconsin

\section{Correspondence}

Gabriel Zayas-Cabán, Department of Industrial and Systems Engineering, University of Wisconsin-Madison,

Madison, WI 53706.

Email: zayascaban@wisc.edu

\section{Present Address}

Gabriel Zayas-Cabán, Department of Mechanical Engineering, 1513 University

Avenue Madison, WI 53706

\section{Funding information}

National Institute of Mental Health, Grant/Award Number: K01MH112876; NIH, Grant/Award Number: R01HL094786-05A1
In emergency departments (EDs), care providers continuously weigh admissions against continued monitoring and treatment often without knowing their condition and health needs. To understand the decision process and its causal effect on outcomes, an observational study must contend with unobserved/missing information and a lack of exchangeability between admitted and discharged patients. Our goal was to provide a general framework to evaluate admission decisions from electronic healthcare records (EHRs). We describe admission decisions as a decision-making process in which the patient's health needs is a binary latent variable. We estimate latent health needs from EHR with only partial knowledge of the decision process (ie, initial evaluation, admission decision, length of stay). Estimated latent health needs are then used to understand the admission decision and the decision's causal impact on outcomes. For the latter, we assume potential outcomes are stochastically independent from the admission decision conditional on latent health needs. As a case study, we apply our approach to over 150000 patient encounters with the ED from the University of Michigan Health System collected from August 2012 through July 2015. We estimate that while admitting a patient with higher latent needs reduces the 30-day risk of revisiting the ED or later being admitted through the ED by over $79 \%$, admitting a patient with lower latent needs actually increases these 30-day risks by $3.0 \%$ and $7.6 \%$, respectively.

\section{KEYWORDS}

causal inference, emergency department admission decisions, latent variables, potential outcomes

\section{1 | INTRODUCTION}

In the United States, hospitalizations account for one-third of healthcare expenditure with over half of admissions originating from the emergency department (ED). ${ }^{1}$ A growing portion of admission decisions to inpatient hospital units are being made in the ED. ${ }^{2}$ By ordering more tests or monitoring patients longer, an ED care provider can delay their admission decision to better inform their final decision, but this can delay treatment to other time-critical patients and lead to long waits in already-overcrowded EDs. Alternatively, patients are being increasingly sent to a medical short-stay or observation units to allow for extended evaluation for up to 24 to 48 hours to better determine if the patient should be sent home or admitted. ${ }^{3,4}$ However, whether delaying or increasing admission decisions improve outcomes, efficiency, or costs, is an open-ended and hotly debated topic with important policy implications. ${ }^{5,6}$ Thus, ED care providers must 
quickly discriminate between who to send home, continue to treat/monitor, send to a short stay unit, or admit to the hospital, balancing patient outcomes with costs and timely access to care for all patients.

The admission decision process in EDs begins when a patient arrives and proceeds to triage, where they are usually assigned an acuity level based on severity of illness. Acuity is commonly assigned using the emergency severity index (ESI), a five-level triage algorithm designed to facilitate the sorting and streaming of patients. Higher acuity patients (1 or 2) are almost immediately brought to a bed for treatment. Lower acuity patients $(3,4$, and 5$)$ wait for treatment until they are brought to a bed. Once in a room/bed, they are visited by providers to determine a plan of care involving a series of examinations, diagnostic testing such as imaging (eg, radiographs, ultrasound CT scans, MRI), laboratory work, and treatment. After testing and treatment, the patient is either well enough to be discharged home or is admitted to the hospital. This seemingly simple decision-making scenario turns out to be surprisingly complex, with important health and policy implications.

Because admission is one of the most expensive routine decisions made in health care for a patient, ${ }^{7}$ it is important to determine how the admission decision causally impacts patient outcomes such as ED revisits and hospital readmissions. Patients or clinics would ideally be randomized to various treatment groups (eg, discharge home, admit), but randomized clinical trials are difficult, if not unethical, to conduct for this question. Alternatively, electronic health records (EHRs) could be used to leverage large amounts of transactional data on daily ED operations, but then finding an association between admission decisions and an outcome may be insufficient to conclude that the decision caused the outcome. The issue is that the decision to admit a patient or not cannot be reasonably assumed to be independent from their potential outcomes, where potential outcomes are those outcomes that would have been observed if each person could be assigned to all treatment groups. Notably, the severity of a patient's condition is expected to strongly influence both admission decisions and outcomes.

Causal inference methods such as propensity score matching, inverse probability weighting, standardization, g-estimation, and instrument variables (IVs) attempt to overcome this issue. To date, most empirical studies on transfer decisions between hospital units, such as admitting a patient to an inpatient hospital unit, use an IV approach. This approach relies on a variable called an instrument that is correlated with the treatment but not with the outcomes except perhaps through its association with treatment. Kim et al ${ }^{8}$ examined how congestion impacts ICU admission decisions and patient outcomes. They used congestion as an IV to identify the causal effect of ICU admission decisions on patient outcomes. Chan et $\mathrm{al}^{9}$ considered admission to ICU versus step down units (SDUs), an intermediate level of care for semi-critically ill patients who are not sick enough to require intensive care but not stable enough to be treated on a general ward. They used IV approaches to estimate the impact on patient outcomes of routing patients to the SDU from the ED as well as the ICU. Kim et $\mathrm{l}^{10}$ studied whether ICU occupancy influences ICU admission decisions and patient outcomes in a retrospective study also using IVs. Bartels et $\mathrm{al}^{11}$ used IVs to address the potential bias in hospital length-of-stay. Causal inference studies on the ED are more rare. One example is the work of Kuntz et al. ${ }^{12}$ The authors also used IVs to support replacing general hospitals with what they term as "value-adding process clinics" and "solution shop hospitals" for less and more complicated patients, respectively. In these applications, researchers justify their use and choice of IVs as a way to overcome potential bias when unobserved patient severity/needs affects both transfer decisions and patient outcomes.

The challenge with IVs is in identifying an appropriate instrument. ${ }^{13-15}$ When there is a strong backdoor factor (also referred to as endogenous variable or confounder), such as we expect patient severity is for ED admissions and outcomes, the instrument should also be strongly correlated with treatment assignments. This can be extremely challenging. Moreover, an IV approach does not model the backdoor factor, unobserved patient severity, or the process upon which a physician accrues information while treating the patient until an admission decision is finally made. Thus, this approach may not offer insight into the actual decision-making process.

Other methods to making causal inferences try to mimic a randomized trial by compensating for the bias introduced by backdoor factors. These methods include propensity score matching, inverse probability weighting, standardization, and g-estimation (see, eg, other works ${ }^{16-19,20-23}$ for comprehensive treatments on these methods). These methods must satisfy certain assumptions: positivity, consistency, and exchangeability, of which exchangeability is arguably the hardest to satisfy. Full exchangeability says that treatment assignments are stochastically independent from potential outcomes. Often too strict, full exchangeability is often replaced with conditional exchangeability, which requires treatment assignments are stochastically independent from potential outcomes within strata (eg, strata defined by sex and age). Additionally, these methods require a conceptual understanding of what factors impact treatment assignments, and this conceptual understanding needs to be formalized mathematically. These methods can fail when an unobserved or latent variable introduces hidden bias that is not taken into account in the model. ${ }^{21}$ Latent variable models within causal inference frameworks have been studied in the literature (see, eg, related works ${ }^{24-32}$ ). In the work of Pearl and Robins, ${ }^{27}$ for instance, the 
authors considered how to causally evaluate a class of probabilistic diagrams when there are unmeasured, or latent, variables. In the work of Louizos et $\mathrm{al},{ }^{32}$ the authors used latent variable models to estimate latent confounders (ie, factors that affect both an intervention and its outcome) and individual-level causal effects from observational data. Applications have been studied in the works of Gadbury et $\mathrm{al}^{29}$ and Bartolucci and Farcomeni. ${ }^{31}$ In the work of Gadbury et al, ${ }^{29}$ for instance, the authors studied weight loss intervention programs. The authors modeled unintentional weight loss as a latent variable and used potential outcomes to estimate associations between weight loss and mortality. Motivated by angiography in myocardial infarction, Bartolucci and Farcomeni ${ }^{31}$ proposed a causal inference method for treatment in a two-arm experimental study with noncompliance in treatment and control arms. Their model includes individual patient covariates and latent variables for unobserved heterogeneity between subjects.

This prior work provides useful models for embedding latent variables in causal inference frameworks. Here, we aim to posit and model a latent variable to capture a care provider's uncertainty related to patient's health needs. Our paper is based on the hypothesis that explicitly modeling a patient's unknown, or latent, health needs can help evaluate admission decisions and estimate causal effects of admissions on outcomes. We introduce a model that describes the admission decision as a decision-making process with health needs as a latent variable. We make the assumption that latent health needs provides a natural conditional exchangeability assumption: admission decisions are stochastically independent from potential outcomes within a group of individuals with similar latent health needs, and integrate this assumption into the potential outcomes framework to estimate causal effects from observational data. We use this approach to examine data on over 150000 patient encounters in the ED and inpatient units from the University of Michigan Health System (UMHS) collected from August 2012 through July 2015.

We view our work as making the following contributions.

1. A continuous-time decision making model of the admission decision process in the ED in which health needs are explicitly captured as a latent variable.

2. A method to estimate causal effects using the latent variable to overcome a lack of exchangeability.

In the process, we impose minimal conditions on EHR data: only an initial observation, final decisions, length of stay, and demographic information are needed, so the method should be applicable to most ED datasets. The rest of the paper is organized as follows. Section 2 describes the modeling framework. Section 3 describes parameter specification and estimation. Section 4 studies estimation via simulation. Section 5 contains our case study and a sensitivity analysis. Section 6 contains a discussion of these results and our conclusions.

\section{ADMISSION DECISION PROCESS}

\section{1 | Decision-making model}

We present a model of the decision process for a single patient from the perspective of a care provider (eg, physician) tasked with making an admission decision. We let random variable $X \in \mathbb{R}^{k}$ denote patient characteristics known prior to the start of treatment such as age and sex. Let $H \in\{0,1\}$ denote the hidden or latent health state. The health state denotes the (unknown) patient's needs for hospital resources (eg, level of treatment). For this study, we assume $H$ is a binary random variable with $H=1(0)$ representing higher (lower) needs. We remark that the results presented here also hold in the case when $H$ can take on a finite number of ordered values.

We let $Z \in\{0,1\}$ be a random variable denoting an initial (noisy) observation, or belief, of health needs taken prior to the start of treatment or testing by the medical provider, where $Z=1(0)$ represents an initial belief of higher (lower) health needs. In practice, observation $Z$ is usually in the form of ESI or acuity level. Lastly, we define random variables $A \in\{0,1\}$ to represent the realized admission decision, ie, admit (1) or discharge (0), and $T \in(0, \infty)$ to represent the time from when treatment begins until the admission decision is made, ie, treatment time. We assume that the random vector $(X, H, Z, A, T, Y)$ are independent and identically distributed, where $Y$ will denote outcomes which we define later.

Putting it all together, immediately after an initial (noisy) assessment, $Z$, the patient undergoes treatment/testing at time $t=0$ that yield new (noisy) observations of the patient's health state. The physician views observations collected so far until enough information is collected after a random amount of time $T$ to decide among two mutually-exclusive decisions: discharge patient home or admit patient to an inpatient hospital unit, with $A=0,1$ denoting, respectively, the decision to discharge or admit. Once the physician decides to admit or discharge a patient, a patient no longer undergoes treatment/testing and waits to be sent home or admitted to the hospital. 


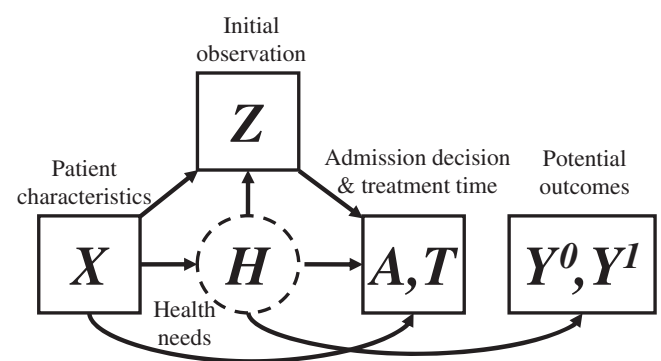

FIGURE 1 Bayesian network of admission decision model. Patient health needs $H$ influences an initial observation $Z$. Care providers use this observation and other observations collected so far from treatment and testing until time $T$, when they make a final decision $A$ on whether to admit or discharge. Dashed circle represents our latent variable, whereas rectangles represent observed variables

Our model of the admission decision process yields the Bayesian network depicted in Figure 1, for which nodes are sets of variables and edges link one node to the other whenever variables in the latter node are conditionally dependent on variables in the former node. For example, we see from the network that the admission decision $A$ depends on the initial observation $Z$, health state $H$, and patient characteristics $X$.

\section{2 | Structural model for admission decision and treatment time}

We introduce a joint structural model for final decision $A$ and treatment time $T$ to reflect the decision-making process of a care provider. It is a drift-diffusion model that captures similar decision-making scenarios, whereby one collects information continuously over time until eventually deciding between two mutually exclusive options after a variable amount of time (see, eg, the work of Krajbich and Rangel ${ }^{33}$ for an application in neuroscience of drift-diffusion models to decision-making). Such an approach is also used in threshold regression to jointly model a continuous time-to-event and an event's outcome. ${ }^{34}$ Specifically, we introduce parameters $b(X), c(X, Z), d(H)$, and construct a joint model of admission decision $A$ and treatment time $T$ (conditional on $X, H$ and $Z$ ) from first-passage locations and times of Brownian motion $B_{t}$ (Figure 2). Assuming a drift of $d(H) b(X)$ and infinitesimal variance of $\sigma^{2}$, then Brownian motion $B_{t}$ is a continuous-time stochastic process with stationary and independent increments $B_{t+s}-B_{t}$, which are normally distributed with mean $d(H) b(X) s$ and variance $\sigma^{2} s$. We model treatment time $T$ as the first-passage time of $B_{t}$ out of an open interval $(0, b(X))$ starting at some point $B_{0}:=c(X, Z) b(X)$ with $c(X, Z) \in(0,1)$, ie,

$$
T:=\inf \left\{t>0: B_{t} \notin(0, b(X))\right\} .
$$

The admission decision $A$ is then captured by whether $B_{t}$ exits through 0 or through $b(X)$, ie,

$$
A:= \begin{cases}0 & B_{T}=0 \\ 1 & B_{T}=b(X) .\end{cases}
$$

Note that the value of $B_{t}$ does not represent a physical quantity. We can thus scale $B_{t}, \sigma$, and $b(X)$ without changing the distribution of $(A, T)$ given $X, H, Z$. Hence, it is without loss of generality that we assume $\sigma^{2}=1$ with units $1 /$ time.

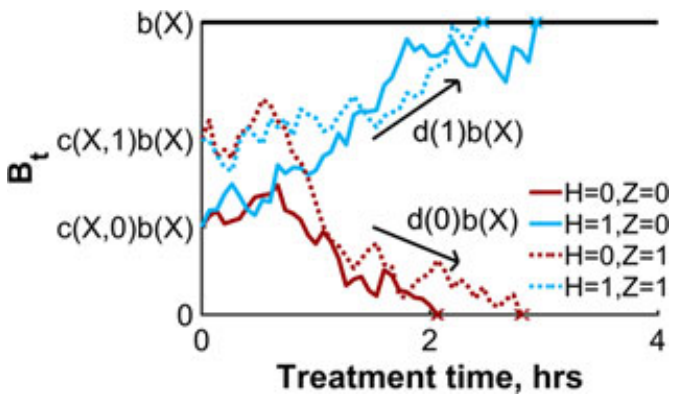

FIGURE 2 Structural model of treatment time $T$ and admission decision $A$ is constructed from, respectively, the first-passage time and exit location of Brownian motion $B_{t}$. To capture the dependence on patient characteristics $X$, latent health needs $H$, and initial observation $Z$, we assume Brownian motion starts at a point $c(X, Z) b(X)$ and drifts at a speed of $d(H) b(X)$ until reaching the boundary 0 (discharge) or $b(X)$ (admit) [Colour figure can be viewed at wileyonlinelibrary.com] 
Based on the assumptions earlier, we arrive at a structural model for the joint density function of $(A, T)=(a, t)$, ie,

$$
\mathbb{P}(T \in[t, t+d t], A=a \mid X, H, Z):=g(a, t \mid b(X), c(X, Z), d(H)) d t,
$$

where the differential $d t$ here is informally used to denote an infinitely small (or infinitesimal) change in value $t$ and $g(a, t \mid b, c, d)$ denotes the joint density of $(A, T)=(a, t)$ for a given initial point $b c$, drift rate $d b$, and upper boundary $b$. Note that, to place these parameter functions into the context of the admission decision-making scenario, we assumed that $c$ is a function of patient characteristics $X$ and initial observation $Z$ to reflect that a medical care provider only has $Z$ and $X$ to initially evaluate the patient. We assumed that the drift term $d$ is a function of health needs $H$ to reflect that information collected by the care provider in the ED will be determined largely by their health needs $H$. Lastly, we assumed that boundary $b$ depends on $X$ to reflect that the level of evidence required to make a decision depends on patient characteristics.

Remark 1. Function $g$ has two equivalent expressions, both in the form of infinite series (see Appendix B). Following the work of Navarro and Fuss, ${ }^{35} \mathrm{~g}$ can be approximated by the truncated version of one series for small $t$ and the truncated version of the other series otherwise. In the aforementioned work, ${ }^{35}$ guidelines are provided for when to use which approximation.

\section{3 | Potential outcomes with latent health needs}

We now consider an outcome of interest, represented by a random variable $Y \in \mathbb{R}$. The outcome random variable $Y$ may or may not depend on other variables in our admission decision process. Common outcomes of interest in the ED include ED revisits (ie, return to the ED within, say, 30 days) and hospital admission within 30,60, or 90 days after the patient is discharged. These two outcomes are often used to measure the quality of hospital care, since it could mean patient follow-up care was not properly organized, or that the patient was not adequately treated before discharge. ${ }^{36,37}$

We want to infer the causal impact of admitting a patient on the outcome of interest. For causal inference, it is common to consider random potential outcomes $Y^{a} \in \mathbb{R}$ for $a=0,1$, where $Y^{a}$ represents the outcome that would have been observed if we always admitted our model patient $(a=1)$ or if we always discharged our model patient $(a=0)$. Hence, we assume actual outcomes $Y$ are either $Y^{0}$ if the patient is discharged $(A=0)$ or $Y^{1}$ if the patient is admitted $(A=1)$, leaving one of the potential outcomes missing.

We want to use actual outcomes $Y$ to provide information on the distribution of potential outcomes $Y^{a}$. If admission decisions were randomized, then potential outcomes $Y^{a}$ would be independent from the admission decision $A$, and $Y^{a}$ would have the same distribution as $\left(Y^{a} \mid A=a\right)=(Y \mid A=a)$. Put differently, with randomized admission decisions, potential outcomes for admitted patients are exchangeable with potential outcomes for discharged patients. In other applications, potential outcomes may reasonably be assumed to be independent from treatment assignments, ie, exchangeability can be a reasonable assumption, even when treatment assignments are not randomized. However, for EDs and other hospital transfer decisions, it should be clear that these decisions cannot be reasonably assumed to be independent from potential outcomes. Admitted patients are necessarily in a poorer health state than discharged patients, and whether a patient is readmitted to an inpatient unit or revisits the ED is likely to be related to a patient's health.

Given this conceptual understanding of the ED admission process, it is then natural to try to account for the impact that severity of patient health has on both admission decisions and outcomes. We address this by exploiting the latent variable $H$ to control for confounding between actual outcomes and admission decisions. Specifically, we make the following assumption.

Assumption 1 (Conditional exchangeability).

Potential outcomes $Y^{a}(a=0,1)$ are independent from the admission decision $A$ conditional on the health state $H$ and patient characteristics $X$.

Remark 2. Conditional exchangeability is a common assumption for causal inference approaches known as standardization and inverse probability weighting (IPW). These approaches assume potential outcomes $Y^{a}$ are independent from the admission decision $A$ conditional on a subset of observed variables $U$ and then use the fact that $Y^{a} \mid U$ has the same distribution as $Y \mid U, A=a$ to estimate $\mathbb{E} Y^{a}$ for $a=0,1$. 


\section{4 | Discussion on the decision-making model}

Model choice is important for causal inference approaches because estimates generally depend on a model that specifies how variables influence treatment assignments and outcomes. The model should be chosen carefully to recover accurate estimates from causal inference approaches. We thus wanted a model to best reflect the actual admission decision process.

We highlight three important features of the decision-making model. First, patient health needs are latent or hidden ( $\mathrm{cf}$ the work of Knott and Bartholomew ${ }^{38}$ ). In the ED, physician's do not always arrive at complete diagnoses for patient symptoms (eg, chest pain, abdominal pain, syncope, headache, shortness of breath) at the end of the visit. As a result, there is variability in how physicians determine the need for hospitalization. Second, patient health needs are elucidated through a series of noisy (ie, imperfect) observations through diagnostic testing and/or treatment. The noisy assumptions are because a physician's decision to admit a patient involves many unobserved complex factors. Moreover, diagnostic testing and response to treatment may be ambiguous. Third, decisions to transfer or to continue treatment and testing occur at continuous and variable times throughout a patient's stay in a hospital unit.

To capture these features, we used a particular version of threshold regression ${ }^{34}$ based on hitting time of a drift-diffusion process on one of two boundaries. This regression model describes processes of evidence accumulation, such as how humans discriminate between two choices over time. ${ }^{39}$ Unlike traditional regression models, it captures many important features: continuous decision times; initial bias in the admission decision process; rate at which information is collected; and the threshold level of information at which point a final decision is made. The underlying assumption is that the individual, in this case, the physician, extracts, per time unit, a constant piece of evidence from the stimulus (drift), which is disturbed by noise (diffusion). This accumulation stops once enough evidence has been sampled and a decision is made. These features are consistent with the admission decision model.

Another consideration is that our model of the decision-making process is not a standard survival model. Despite being nonstandard, threshold regression has been used to model survival processes for many applications, including hospital length of stay and latent health status. ${ }^{34,40-42}$ In fact, the more commonly-used inverse Gaussian distribution is also a threshold regression model. See the work of Lee and Whitmore for an overview and examples. ${ }^{34}$ Moreover, our model also simplified analysis in three main regards. First, we could use one joint model for both the admission decision and treatment time. By contrast, a more conventional survival analysis model (eg, Weibull regression model) would require two regression models, one model for the admission decision, and another for treatment time conditional on the admission decision or one model for the treatment time, and another for admission decision conditional on the treatment time. Second, if we were to use two regression models as discussed earlier, we would require 2 additional parameters to capture main effects (characteristics, acuity, and health state in both models and treatment time or admission decision in the second model) compared to our model.

Third, there was a simple way to capture the dependence of the admission process $(A, T)$ on the initial observation $Z$ and health state $Z$ because our model was a more realistic model of the admission process than other conventional survival models. The initial observation $Z$ influences the initial level of evidence, and the health state $H$ influences the rate of evidence accumulation. These assumptions can automatically capture treatment times that are longer on average when there was a mismatch between acuity and admission decision, which we observe in our dataset. By contrast, capturing this effect using the two-model approach discussed earlier would require interaction terms in our regression model, leading to even more coefficients to estimate.

\section{3 | PARAMETER SPECIFICATION AND ESTIMATION}

To fit the admission decision model to EHR data, we assume that we have data on $N$ visits and that, for visit $n$ ( $n=$ $1,2,3, \ldots, N)$, the following variables are collected: a vector of patient characteristics $x_{n} \in \mathbb{R}^{k}$; an initial assessment $z_{n} \in\{0,1\}$; admission decision $a_{n} \in\{0,1\}$; treatment time $t_{n} \in(0, \infty)$; and a binary outcome $y_{n} \in\{0,1\}$. Although we focus on binary outcomes, results can be extended to continuous outcomes. We assume that the admission decision process is independent and identically-distributed for each visit.

Along with parameter functions $b(X), c(X, Z), d(H)$, we specify the admission decision process with parameter functions

$$
\alpha(X):=\mathbb{P}(H=1 \mid X) \quad \text { and } \quad \beta(X, H):=\mathbb{P}(Z=1 \mid X, H) .
$$

We further restrict attention to when

$$
\operatorname{logit} \alpha(X), \operatorname{logit} \beta(X, H), \log |d|(H), \operatorname{logit} c(X, Z), \log b(X)
$$


are linear in their arguments and estimate the set of linear coefficients of these functions. For $k$-dimensional $X$, this restriction leads to $8+4 k$ unknown parameters. The sign of the drift rate $d(H)$ is assumed to be negative for lower health needs $(H=0)$ and positive for higher health needs $(H=1)$ to capture propensity for lower health needs patients to be discharged and higher health needs patients to be admitted. For outcomes, we assume potential outcomes depend linearly on latent health needs, ie,

$$
\begin{aligned}
\mathbb{P}\left(Y^{a}=1 \mid X, Z, T, H\right) & =\mathbb{P}(Y=1 \mid X, Z, T, A=a, H) \\
& :=\mu_{1}+\left(\mu_{2}-\mu_{1}\right) H+\left(\mu_{3}-\mu_{1}\right) a+\left(\mu_{4}-\mu_{2}-\mu_{3}+\mu_{1}\right) H a,
\end{aligned}
$$

so that

$$
\mathbb{E}\left[Y^{1}-Y^{0} \mid H=0\right]=\mu_{3}-\mu_{1} \text { and } \mathbb{E}\left[Y^{1}-Y^{0} \mid H=1\right]=\mu_{4}-\mu_{2},
$$

where we employ the conditional exchangeablility Assumption 1 to relate potential outcomes to actual outcomes. Potential outcomes lead to an additional 4 unknown parameters, leading to $12+4 k$ unknown parameters in total. Note that the latent variable $H$ represents a confounder in the sense that it partitions the population into strata such that, within each stratum, exchangeablity of the $A=0$ and $A=1$ populations holds, whereas exchangeability might not hold marginally over $H$. The latent variable $H$, however, may also serve as a modifier of the treatment effect of $A$. Effect modification occurs when the interaction term $\left(\mu_{4}-\mu_{2}-\mu_{3}+\mu_{1}\right)$ is nonzero. Furthermore, we chose to omit variables $X, Z, T$ in the aforementioned expression for potential outcomes to reduce the number of parameters and to reflect that patient characteristics, acuity, and treatment time might not have a direct effect on potential outcomes when controlling for patient health needs. However, our framework is sufficiently flexible that variables $X, Z, T$ may be included if desired, and confidence intervals of any resulting coefficients may be checked to see if they contain 0 to justify removing variables.

Let $\theta$ be the vector of unknown parameters and $\Theta:=\mathbb{R}^{12+4 k}$ be the set of possible parameter vectors. We estimate model parameters by performing maximum likelihood estimation (MLE) using the expectation-maximization (EM) algorithm..$^{43}$ For the EM algorithm, we need to specify the complete data likelihood of observing $(H, Z, A, T, Y)=(h, z, a, t, y)$ given parameters $\theta$ and patient characteristics $X=x$. Based on our Bayesian network, the complete log-likelihood is expressed as

$$
\begin{array}{r}
(1-a)\left(y \log \left[\mu_{1}(1-h)+\mu_{2} h\right]+(1-y) \log \left[\left(1-\mu_{1}\right)(1-h)+\left(1-\mu_{2}\right) h\right]\right)+ \\
a\left(y \log \left[\mu_{3}(1-h)+\mu_{4} h\right]+(1-y) \log \left[\left(1-\mu_{3}\right)(1-h)+\left(1-\mu_{4}\right) h\right]\right)+ \\
\log g(a, t \mid b(x), c(x, z), d(h))+z \log \beta(x, h)+(1-z) \log [1-\beta(x, h)]+\ldots \\
h \log \alpha(x)+(1-h) \log [1-\alpha(x)],
\end{array}
$$

where we suppressed the dependence on $\theta$ in our parameter functions.

Confidence intervals are estimated using a numerical approximation to Oakes Identity ${ }^{44}$ used in EM methods to estimate Fisher's information matrix, which, when inverted, yields estimates of sampling variances for each parameter. These variances were then used to construct $95 \%$ confidence intervals assuming parameter estimates are normally distributed. Confidence intervals for functions of parameter estimates were estimated using the delta method. For completeness, we consider an alternative estimation approach in the Appendix that first estimates parameters for the admission decision process and then estimate parameters for outcomes. This alternative approach was considered to mirror our dual goals of discovering how a decision process could be explained by latent health needs and then determining how outcomes are influenced by this latent variable; and because estimating latent variables in other settings separately from effects of latent states had also been suggested in the work of Bolck et $\mathrm{a}^{45}$ and shown numerically in the work of Dziak et al ${ }^{46}$ to be more robust to model violations. This alternative approach also does not require distribution assumptions on outcomes.

\section{4 | SIMULATION}

We consider two simulation examples to assess parameter estimation and robustness of our estimates to violations in the conditional exchangeability Assumption 1. We compared mean difference in potential outcomes $\mathbb{E} Y^{1}-\mathbb{E} Y^{0}$ for our method to three other methods. Inverse probability weighting was performed by building a logistic regression model to predict admission decisions from patient characteristics $X$, initial observation $Z$, and treatment time $T$. We then estimated mean potential outcomes $\mathbb{E} Y^{a}$ by taking a weighted average of outcomes $Y$ with admission decision $A=a$, where weights are given by one over the probability of admission decision $A=a$ predicted from the logistic regression model. The method of g-estimation was performed by building a logistic regression model to predict admission decision from $X, Z, T$, and a 
variable $J:=Y-\psi A$ for some parameter $\psi$. We varied $\psi$ until the fitted regression model had a zero coefficient for $J$. In order to assess the value of using a latent-variable to adjust for confounding, the last method we compared estimated mean potential outcomes from our model when the dependence on the latent variable $H$ is removed. Specifically, we dropped the dependence on $H$ in the initial observation $Z$, admission decision process $(A, T)$, and Assumption 1 . We then adjusted the $\log$ drift term $\log |d|(H)$ to be linear in the initial observation $Z$ rather than $H$ and adjusted to the logit of potential outcomes $\operatorname{logit} \mathbb{P}\left(Y^{a} \mid X, Z, T\right)$ to be linear in patient characteristics $X$ and initial observation $Z$ rather than $H$. Throughout this section, we assume patient characteristics $X$ is a two-dimensional random vector $\left(X_{1}, X_{2}\right)$ with $X_{1}$ describing a binary characteristic such as gender and $X_{2}$ describing a numerical variable such as age.

\section{1 | Example 1: correct model}

We simulated $N$ ED visits by sampling directly from the model of admission decisions and outcomes for a particular choice of 20 unknown parameters (see Table A1 in Appendix A). We considered both $N=1000$ and $N=10000$. For comparison, our UMHS dataset has over 150000 visits. Parameters were estimated along with their confidence intervals and the process repeated for a total of 500 replicates. To arrive at reasonable parameters, we used our UMHS dataset with the admission decisions as a proxy for health needs (see Table 3 for the description of the UMHS dataset and see Appendix D for justification of parameter choices).

For this model, we checked bias, mean squared error (MSE), and coverage for parameter estimates. Results are summarized in Table A1 in Appendix A. When $N=1000$, certain parameters are not estimated accurately. For example, the linear coefficient for the term $H=1$ in logit $\beta(X, H)$ is estimated poorly in terms of bias (1.6) and MSE (44.4). Confidence intervals, however, capture this uncertainty with $94 \%$ coverage of the true parameter. We also understate estimated outcomes for higher needs patients who are discharged $\left(\mu_{2}\right)$ by about $12 \%$. In this case, confidence intervals are associated with only $90 \%$ coverage. All estimates, however, improve significantly in terms of bias, MSE, and coverage when $N$ increases to 10000 data points. Based on this, we hypothesize that estimates will improve when we increase the number of data points to 150000 .

Out of all of the parameters, $\mu_{2}$ was the most difficult to estimate. MSE decreased from 0.0801 for 1000 data points to 0.0130 for 10000 data points, and we expect even more accurate estimates for $\mu_{2}$ when the number of data points is increased to $N=150000$. This parameter corresponds to higher-needs patients that are discharged (ie, $H=1$ and $A=0$ ). It was estimated from discharged patients, but, by design, the majority of these patients have lower health needs $(H=0)$. By our choice in parameters, this group tended to be the smallest group (out of the four groups divided on health needs and admission decision) in simulated data samples $(\approx 5 \%)$, which may explain why it is the most difficult group for which to estimate outcomes.

To connect to other causal inference methods, we also estimated mean difference in potential outcomes

$$
\mathbb{E}\left[Y^{1}-Y^{0}\right]
$$

and log odds ratio of potential outcomes

$$
\log \frac{\mathbb{E} Y^{1} \mathbb{E}\left[1-Y^{0}\right]}{\mathbb{E} Y^{0} \mathbb{E}\left[1-Y^{1}\right]} .
$$

Mean difference in potential outcomes has an actual value of -0.12 for this example (Table 1 ). When $N=1000$, our estimates for this quantity had a bias of 0.019 , MSE of 0.014 , and $86 \%$ coverage. When $N=10000$, estimates for this quantity improve leading to a bias of 0.0080 , MSE of 0.0017 , and $90 \%$ coverage. Log odds ratio of potential outcomes has an actual value of -0.63 . When $N=1000$, our estimates for this log odds ratio had a bias of 0.067 , MSE of 0.46 , and $86 \%$ coverage. When $N=10000$, our estimates perform better with a bias of 0.041 , MSE of 0.049 , and $92 \%$ coverage.

TABLE 1 Performance of marginal estimates when the model is correct (Example 1) in terms of bias, mean square error (MSE), and percent coverage of true parameters for $95 \%$ confidence intervals. True parameter values are also reported. Variable $X_{2}$ was uniformly distributed between $-1 / 2$ and $1 / 2$

\begin{tabular}{lccccrrrr} 
& & \multicolumn{4}{c}{$\mathbf{N}=1000$} & \multicolumn{3}{c}{$\mathbf{N}=\mathbf{1 0 0 0 0}$} \\
& & Value & Bias & MSE & Coverage & Bias & MSE & Coverage \\
$\mathbb{E}\left[Y^{1}-Y^{0}\right]$ & $\mathrm{n} / \mathrm{a}$ & -0.12 & 0.0192 & 0.0143 & 0.86 & 0.008 & 0.0017 & 0.90 \\
$\log \frac{\mathbb{E} Y^{1} \mathbb{E}\left[1-Y^{0}\right]}{\mathbb{E} Y^{0} \mathbb{E}\left[1-Y^{1}\right]}$ & $\mathrm{n} / \mathrm{a}$ & -0.63 & 0.0674 & 0.4608 & 0.86 & 0.0411 & 0.0491 & 0.92 \\
\hline
\end{tabular}


By comparison, the other methods (IPW, g-estimation, and fitting our model without the latent variable) led to poor estimates of mean difference in potential outcomes, which do not improve with sample size. For instance when $N=1000$, IPW led to an average estimate of mean difference in potential outcomes of 0.053 far from the true value of -0.12 . When $N=1000$, IPW led to a bias in mean difference in potential outcomes 0.173 and MSE of 0.031 , whereas g-estimation led to a bias of 0.166 and MSE of 0.028 and fitting our model without the latent variable led to a bias of 0.176 and MSE of 0.032 . When $N=10000$, IPW led to a bias in mean difference in potential outcomes of 0.168 and MSE of 0.028 , whereas g-estimation led to a bias of 0.161 and MSE of 0.026 and fitting our model without the latent variable led to a bias of 0.176 and MSE of 0.031 . Critically, all three methods incorrectly suggest admitting a patient carries a higher risk than discharging a patient. These methods are effectively missing the large risk associated with discharging individuals with higher health needs.

\section{2 | Example 2: violation in conditional exchangeability assumption}

We also checked the robustness of our model to violations in our conditional exchangeability Assumption 1. Keeping the rest of the model and parameters as in Example 1, we introduced an independent Bernoulli random variable $U$ with "success" probability 0.05 that influenced the admission decision and the outcome. If $U=1$ and $A=1$, so that the patient would be admitted, we then redefined $A$ to be zero. For these same patients, who also had higher health needs, we set $Y$ to be one. This models the situation when an admitted patient goes home against medical advice and then comes back to the ED if they originally had higher health needs. Results are summarized in Table 2.

Compared to Example 1, we find that many of the estimates related to latent health needs are more greatly biased. For example, we underestimate the proportion $\alpha(X)$ of higher needs patients. It is important to note that our latent variable is

TABLE 2 Performance of parameter estimates when the Conditional Exchangeability Assumption is violated (Example 2) in terms of bias, mean square error (MSE), and percent coverage of true parameters for 95\% confidence intervals. True parameter values are also reported. Variable $X_{2}$ was uniformly distributed between $-1 / 2$ and $1 / 2$

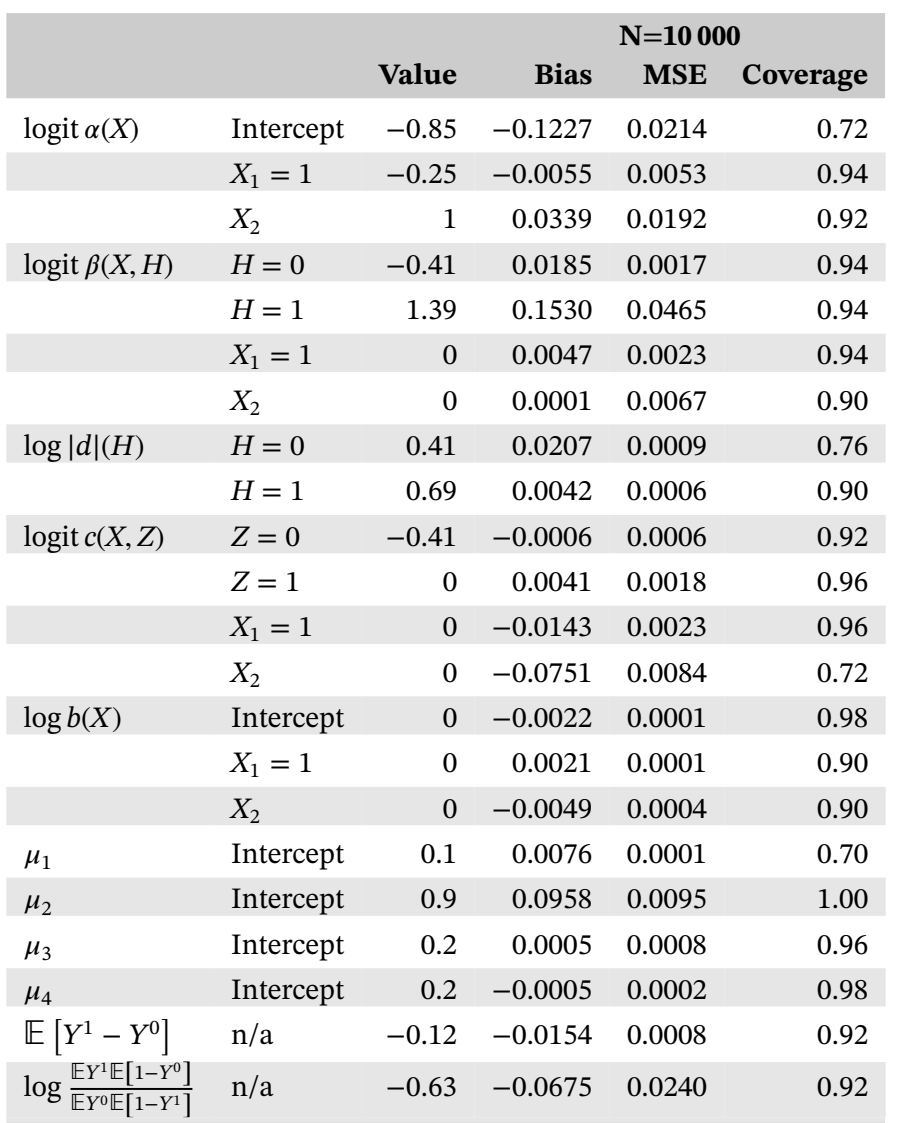


simply a construct to help us understand how care providers in the ED make admission decisions and cannot be measured. Relations between the latent variable and the (measured) variables are specified in a mathematical model to help us explain the statistical properties of the measured variables in terms of the hypothesized latent variable. The implication for Example 2 is that, when multiple endogenous variables share similar relationships between variables (eg, admission decision and outcomes), then the latent-variable tries to reflect both endogenous variables. Thus, we suspect that this bias reflects that our latent variable captures both the health state $H$ and the second endogenous variable $U$.

Although our estimates for the latent variable change, we found that we can achieve similar performance to Example 1 for our estimates of mean difference in potential outcomes and log odds ratio of potential outcomes in which the effect of the latent variable is averaged out. We assume true values are the mean difference in potential outcomes and log odds ratio of potential outcomes conditional on $U=0$, ie, ignoring the effect of $U$ that is not captured by our model. Our estimate for mean difference in potential outcomes has a bias of -0.0154 , MSE of 0.0008 , and $92 \%$ coverage. Our estimate for log odds ratio of potential outcomes had a bias of -0.0675 , MSE of 0.024 , and $92 \%$ coverage.

As in Example 1, the three other methods led to significantly worse estimates of risk difference. Mean difference in potential outcomes has a bias of 0.152 and MSE of 0.023 for IPW, whereas g-estimation led to a bias of 0.142 and MSE of 0.020 and fitting our model without the latent variable led to a bias of 0.162 and MSE of 0.026. Again, both inverse probability and g-estimation incorrectly suggest that admitting a patient carries a higher risk than discharging a patient.

\section{CASE STUDY}

\section{1 | Data}

Patient visits to the ED were analyzed using EHR from the University of Michigan Health Systems (UMHS). For each patient visit, we recovered Demographic information (age, sex); Acuity (ESI); Treatment Time (duration between when treatment starts and ends); Admission Decision (the decision to discharge patient or admit to them to inpatient unit); ED Revisit (a binary outcome [yes/no] specifying whether patient returns to the ED within 30 days of being discharged from any hospital unit, including the ED); and Readmission (a binary outcome [yes/no] specifying whether patient is admitted in the ED to an inpatient unit within 30 days of being discharged from any hospital unit, including the $E D$ ). Note that we used this broader definition of readmission to be able to define a readmission variable for each visit.

For the analysis, we only included patient visits that met the following criteria: treatment start fell within a three-year period between August 1, 2012, and August 1, 2015; the patient was assigned an acuity level of 2 or 3; and the patient was not admitted to an ICU. The latter two criteria were imposed to focus on patient visits with the highest degree of medical uncertainty with respect to needs for longer term acute care need in an inpatient hospital need. A total of 156720 visits were included in the analysis.

A summary of the data is in Table 3 . Note that each sex, age, and acuity group is well represented in the data. Among the patients included in our data set, there are also a reasonable number of patients that are admitted (approximately 29.4\%) and that return to the ED within 30 days after being discharged (approximately 18.7\%). The least represented variable is the number of patients that are admitted within 30 days of being discharge from a hospital unit (approximately 7.1\%).

TABLE 3 Composition of UMHS dataset

( $N=156,720)$ by demography (age, sex), variables related

to ED visit (acuity, treament time, admission decision),

and outcomes (30-day ED revisits and readmissions)

\begin{tabular}{lcc} 
Variable & Count (\%) & Mean (SD) \\
Female & $86814(55.4)$ & \\
Age, years & & $47.7(19.7)$ \\
Acuity 2 & $76033(48.5)$ & \\
Treatment time, hours & & $5.4(4.1)$ \\
\hline Admitted & $46060(29.4)$ & \\
Revisited ED & $29333(18.7)$ & \\
Readmitted & $11176(7.1)$ & \\
\hline
\end{tabular}


Finally, we must contend with missing data. Of the 156720 visits, $91(0.06 \%)$ had missing data; $58(0.04 \%)$ had a missing treatment time; and $33(0.02 \%)$ had a missing admission decision. To handle missing data, we imputed missing variables from one ED visit with corresponding variables of its nearest "neighbor" (ED visit) with complete data in terms of Euclidean distance. ${ }^{47}$ All variables were used for imputation. Age and treatment time were standardized prior to measuring distance in imputation by centering around their respective mean and dividing by their standard deviation. Standardized age was also used in subsequent regression models. Age had a mean of 47.7 years and standard deviation of 19.7 years.

\section{2 | Descriptive statistics of admission decisions and treatment times}

We first analyzed treatment times and admission decisions directly to generate questions about latent health needs (see Table 4). Sex, age, and acuity are important factors in the admission decision process. Men are admitted at a higher rate than women, suggesting that either admission decisions are biased toward men or that women are more likely to visit the ED with lower latent health needs. The latter would explain why women are more likely to visit the ED (see Table 3). Our admission decision model will help us determine if men and women differ on average for similar latent health needs. Similarly, older age groups are admitted at higher rates than younger age groups, which again could suggest bias or differences in latent states at the onset. As for acuity, acuity 2 patients are admitted at higher rates than acuity 3 patients, which is to be expected. It would be natural to attribute these differences to differences in latent health needs.

Analyzing treatment times and admission decisions together further generated questions about latent health needs while demonstrating that understanding the admission decision process requires working with complex relationships. Treatment times are similar between men and women who are discharged but differ between men and women who are admitted. Meanwhile, treatment time is similar among age groups who are admitted, but differ among age groups who are discharged. We question whether identifying health needs is more or less difficult in different age and gender groups. Importantly, treatment time decreases with patient acuity among discharged patients but increases with patient acuity among admitted patients. Are care providers keeping patients longer for whom there is greater uncertainty about their latent health needs? In sum, modeling health needs helps one answer these questions on whether differences in admission rates and treatment times can be attributed to differences in latent health needs.

\section{3 | Fitting the decision-making model to data and estimating causal effects}

We fitted the decision-making model and outcomes described in Section 2 to data on age, sex, acuity, admission decisions, and treatment times using the same procedure described in Section 3. Sex and age were considered to be patient

TABLE 4 Admission rates and treatment times by sex, age, and acuity

\begin{tabular}{|lrrr|}
\hline & & \multicolumn{2}{c}{ Mean (SD) treatment time, hr } \\
Variable & Admitted (\%) & Discharged & \multicolumn{1}{c|}{ Admitted } \\
\hline Male & $22961(32.9)$ & $5.6(4.4)$ & $4.9(3.3)$ \\
\hline Female & $23099(26.6)$ & $5.6(4.3)$ & $5.2(3.4)$ \\
\hline Age & & & \\
\hline $18-24$ & $2702(11.0)$ & $4.6(3.0)$ & $5.0(3.6)$ \\
\hline $25-34$ & $3835(15.0)$ & $5.0(3.4)$ & $5.1(3.4)$ \\
\hline $35-44$ & $4499(20.6)$ & $5.7(4.3)$ & $5.2(3.4)$ \\
\hline $45-54$ & $7251(28.9)$ & $6.3(5.2)$ & $5.2(3.5)$ \\
\hline $55-64$ & $10096(40.2)$ & $6.4(5.2)$ & $5.1(3.4)$ \\
\hline $65-74$ & $9105(50.3)$ & $6.3(5.1)$ & $5.0(3.3)$ \\
\hline $75+$ & $8571(52.3)$ & $5.9(4.3)$ & $5.0(3.1)$ \\
\hline Acuity 3 & $14453(17.9)$ & $5.0(3.6)$ & $5.6(3.6)$ \\
\hline Acuity 2 & $31607(41.6)$ & $6.5(5.2)$ & $4.8(3.2)$ \\
\hline
\end{tabular}



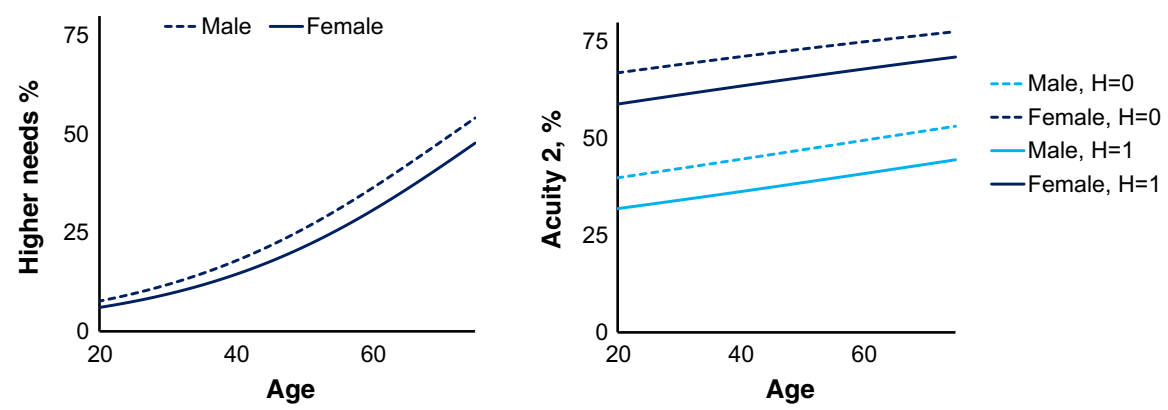

FIGURE 3 Results from parameter estimation: percent with higher latent health needs $H=1$ (determined by $\alpha$ ) and percent with higher initial observation (acuity 2) by latent health needs (determined by $\beta$ ) [Colour figure can be viewed at wileyonlinelibrary.com]

characteristics $X$. Estimates and confidence intervals are reported in Appendix E (Tables E1 and E2). For comparison, we also estimated causal effects by applying IPW and g-estimation approaches using the same approach outlined in Section 4.

\subsection{Inferences from the decision-making model}

In fitting the decision-making model, we can use a patient's latent health needs $(H)$ to gain insight into the admission decision process. For this study, we estimated that, on average, about $25.3 \%$ of patients have higher health needs. Furthermore, men were more likely to have higher health needs than women (Figure 3; parameter estimates and $95 \%$ confidence intervals are in Table E1 of Appendix E). Based on the estimate of the probability $\alpha(X)$ of having higher health needs, about $23.4 \%$ of men had higher health needs at the mean age of 47.0 years, compared to only $19.2 \%$ of women, a difference of about $4.2 \%$. This result could largely explain the difference of $5.6 \%$ in admission rates between men and women. Similarly, we estimated that older individuals are dramatically more likely to have higher health needs than younger individuals. Only $6.2 \%$ of individuals at 18 years of age are estimated to have higher health needs, compared to $44.6 \%$ of individuals at 70 years of age, a difference of $38.4 \%$. Again, a difference in health needs can largely explain the difference of $41.3 \%$ in admission rates between the 18 and 24 year-old group and the 75+ year-old group. Of note, though, individuals with higher health needs are smaller in percentage than admitted patients, indicating that individuals with lower health needs are often admitted.

An individual's health needs is estimated to disagree with the initial observation, which in the UMHS ED is acuity level, about $25 \%$ to $55 \%$ of the time, as determined from the estimate of $\beta(X, H)$ (Figure 3). While this percent mismatch may seem high, it is important to note that acuity level does not only target an individual's health needs but also targets urgency and splits individuals nearly equally between acuity 2 and acuity 3. Mismatch is higher for lower health needs, about $41.6 \%$ compared to $31.4 \%$ for higher health needs at the mean age of 47.0 years. In other words, acuity level is more sensitive to higher health needs than specific.

The final set of parameters $b, c, d$ describes the admission decision process and its dependence on age, sex, health needs, and initial observation. Parameters $b, c$, and $d$ are interpreted, respectively, as the threshold of evidence before a final decision is made, the initial level of evidence acquired from patient characteristics and the initial observation, and the rate at which evidence is accrued for the admission decision. Since these parameters indirectly determine the final decision and treatment time, we briefly discuss their estimates. The initial level of evidence is generally below the midpoint of 0.5 and increases significantly with acuity. This result can be interpreted as follows: a care provider tends at the onset toward the decision to discharge a patient over admitting, particularly acuity 3 individuals. This tendency to discharge a patient is slightly higher in men and younger individuals. We also find that the threshold of evidence is significantly higher for women than men and for older age groups, which can be interpreted as care providers are more careful when making a decision with women and older individuals. Lastly, the rate of evidence is determined by an individual's health needs, with information on higher health needs more quickly accrued relative to lower health needs.

Parameters $b, c, d$ on the admission decision process are best examined by assessing their influence on admission rates and treatment times. Figure 4 depicts how admission rates and treatment times are related to health needs, age, and sex. We estimate that care providers take longer with patients with lower health needs than higher health needs. Care 
Lower needs $(\mathrm{H}=0)$
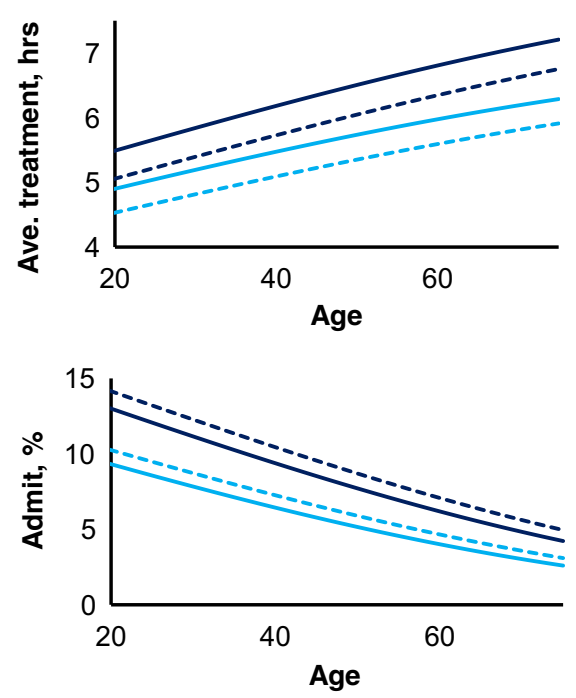

- Acuity 2, Female -- Acuity 2, Male
Higher needs $(\mathrm{H}=1)$
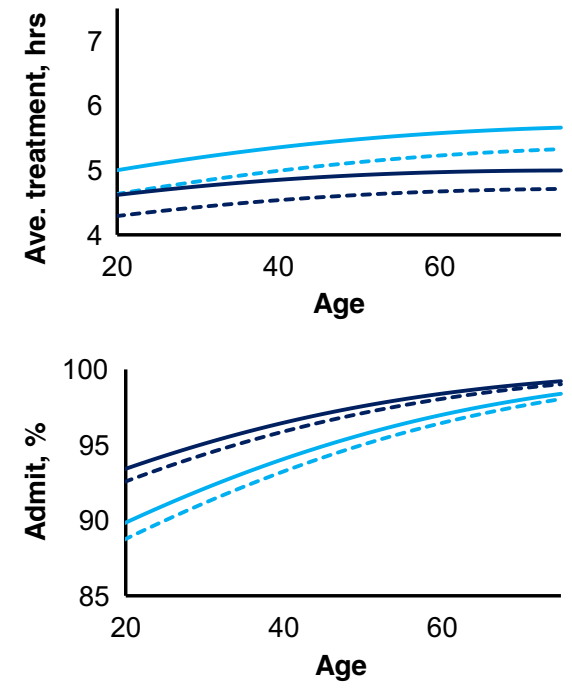

- Acuity 3, Female

-- Acuity 3, Male

FIGURE 4 Estimated average treatment times and admission rates by sex, age, acuity, and latent health needs. Higher health needs is accompanied by shorter treatment times and better accuracy in the final decision compared to a lower health state [Colour figure can be viewed at wileyonlinelibrary.com]

providers also take longer when a patient's initial observation does not match their latent health state. Therefore, for example, care providers spend the most time with lower-needs individuals who are assigned an acuity 2 . This result suggests that care providers keep patients longer when there is uncertainty in their needs. We also estimate that, while the admission decision tends to agree with health needs, lower-needs individuals have greater disagreement with their ideal final decision (which should be to discharge) than higher-needs individuals (which should be to admit). For example, the proportion of lower-need individuals $(H=0)$ who are discharged ranges from $87 \%$ to $97 \%$ depending on acuity, sex, and age, compared to $89 \%$ to $99 \%$ for higher-needs individuals $(H=1)$ who are admitted. Greater disagreement in a lower health state may also translate into keeping patients longer.

As for differences in age and sex, we find that women are kept slightly longer than men and older individuals are kept longer than younger individuals. Put differently, differences in treatment time observed in age and gender groups cannot be attributed solely to health needs, highlighting potential bias in the admission decision process. Importantly, we find that keeping women and older individuals longer is accompanied by greater agreement in their final admission decisions, independent of health needs or acuity level. At best, certain higher-needs individuals are correctly admitted $99 \%$ of the time. At worst, certain lower-needs individuals are incorrectly admitted $13 \%$ of the time.

\subsection{Impact of latent health state on ED revisits and readmissions}

We estimate that admitting an individual in the lower latent state increases the risk of ED revisits and readmissions (Figure 5; Tables E1 and E2 in Appendix E). With lower health needs $(H=0)$, an individual has an estimated 21.6\% $(20.1 \%, 23.3 \%)$ risk of revisiting the ED within 30 days of being discharged if they were admitted, compared to only a $17.3 \%(17.1 \%, 17.5 \%)$ risk if they were discharged, leading to an estimated risk difference of $4.3 \%$. These individuals also have a $10.6 \%(9.1 \%, 12.3 \%)$ risk of being admitted though the ED within 30 days after being discharged if they were admitted, compared to $3.0 \%(2.9 \%, 3.1 \%)$ if they were discharged, leading to an estimated risk difference of $7.6 \%$. Admitting a patient would lead to worse outcomes is contrary to a common assumption that increasing one's level of care means better care.

Admitting an individual with higher health needs $(H=1)$, however, has an opposite effect, significantly decreasing the risk of ED revisits and readmissions (see Figure 5; Tables E1 and E2 in Appendix). With higher health needs, an individual has an estimated $20.1 \%(19.6 \%, 20.6 \%)$ risk of revisiting the ED within 30 days of being discharged if they were admitted, 

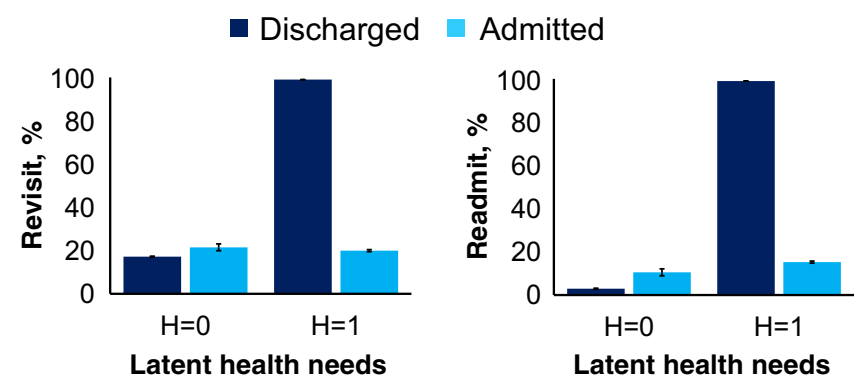

FIGURE 5 Risk of ED revisits and readmissions as a function of admission decisions and latent health needs $H=0$ and $H=1$ [Colour figure can be viewed at wileyonlinelibrary.com]

compared to $100.0 \%(100.0 \%, 100.0 \%)$ risk if they were discharged, leading to a risk difference of about $-79.9 \%$. These individuals also have a $15.4 \%(14.9 \%, 15.8 \%)$ risk of being admitted though the ED within 30 days after being discharged if they were admitted, compared to $99.9 \%(99.5 \%, 100.0 \%)$ if they were discharged, leading to an estimated risk difference of $-84.5 \%$. In other words, discharging an individual with higher health needs carries a significant risk.

Marginalizing over the latent health state, we find that an admission carries a lower risk than a discharge, with an estimated risk difference of $-17.0 \%(-18.1 \%,-15.8 \%)$ for revisits and $-15.6 \%(-16.8 \%,-14.4 \%)$ for readmission. In contrast, IPW and g-estimation conclude that admission carries a higher risk than a discharge. Inverse probability weighting estimates a $20.3 \%$ risk of ED revisits when admitting a patient compared a 18.4\% risk when discharging a patient (risk difference of $1.9 \%$ ), and a $13.7 \%$ risk of readmission when discharging a patient compared to a $4.6 \%$ risk of readmission (risk difference of 9.1\%). G-estimation estimates similar risk differences: 1.5\% higher risk of ED revisit and 9.0\% higher risk of readmission when admitting a patient over discharging a patient. These estimates would suggest that care providers reduce admissions. If, however, our estimates are correct, then these alternative methods miss the high risk of discharging a high-needs patient.

\section{6 | Sensitivity to conditional exchangeability assumption}

We tested how sensitive our estimates of potential outcomes are to violations in the conditional exchangeability Assumption 1. In general, the probability density function of $Y, A, T$ given $H, X, Z$ decomposes as

$$
\left[f\left(A=1, T \mid H, X, Z, Y^{1}\right) \mathbb{P}\left(Y^{1} \mid H, X, Z\right)\right]^{A}\left[f\left(A=0, T \mid H, X, Z, Y^{0}\right) \mathbb{P}\left(Y^{0} \mid H, X, Z\right)\right]^{1-A},
$$

where we use $f$ to denote a general density function. Assumption 1 allows us to drop the dependence of the admission decision process $A, T$ on potential outcomes $Y^{0}, Y^{1}$. Alternatively, if we suppose that

$$
\begin{aligned}
& f\left(A, T \mid H, X, Z, Y^{1}\right) \propto \psi_{1}^{A Y^{1}} f(A, T \mid H, X, Z) ; \\
& f\left(A, T \mid H, X, Z, Y^{0}\right) \propto \psi_{0}^{(1-A) Y^{0}} f(A, T \mid H, X, Z),
\end{aligned}
$$

then violations in the assumption can be captured by choosing values for $\psi_{1}$ and $\psi_{0}$ that are not both one.

We thus fit the model to data for $\psi_{0}$ and $\psi_{1}$ set to either $0.95,0.975,1,1.025$, or 1.05 in a factorial design, resulting in 25 comparisons. In all 25 comparisons, we found that estimates on potential outcomes $\left(\mu_{1}, \mu_{2}, \mu_{3}, \mu_{4}, \mathbb{E} Y^{1}-\mathbb{E} Y^{0}\right)$ for either ED revisits or ED readmissions did not differ from their reference value when $\psi_{0}=\psi_{1}=1$ by more than 0.022 (Tables E3 and E4). For example, estimates of mean difference in potential outcomes ranged from -0.144 when $\psi_{0}=\psi_{1}=0.95$ to -0.168 when $\psi_{0}=\psi_{1}=1.05$ in the case of ED readmissions. Thus, these important causal effects were relatively insensitive to the specified violations in Assumption 1.

\section{6 | DISCUSSION}

We aimed to provide a general framework to evaluate the admission decision process in the ED and to establish causation between admission decisions and outcomes. Our contribution is two-fold: a conceptual model for the ED admission 
decision process in which a patient's health or needs for resources is latent and a causal inference approach that uses latent health state and observational data to determine to what extent admitting a patient improves outcomes. We evaluated our framework with simulation and with an extensive dataset of over 150000 patient encounters in the ED from the University of Michigan Health System collected from August 2012 through July 2015. By modeling latent health needs, we could examine variation in the admission decision process due to latent health needs. We could also estimate separate risks of an ED revisit or readmission for individuals with lower needs versus individuals with higher needs.

Our causal inference approach is based on the potential outcomes framework ${ }^{48}$ and accounts for the lack of independence between treatment assignment and potential outcomes that arises in an observational study (ie, when treatment assignments are not random). More broadly, in an observational study, potential outcomes from one treatment group are not exchangeable with potential outcomes from another treatment group. A popular strategy is to estimate mean potential outcomes within certain groups or strata, for which it is reasonable to assume potential outcomes are exchangeable between treatment groups. ${ }^{20-23}$ Our approach is similar by estimating mean potential outcomes within similar health needs but differs by using strata that are latent.

By marginalizing over the latent variable, we could still recover estimates of the mean difference in potential outcomes, a common target for causal inference approaches. In simulation, we demonstrate that our method accurately estimates mean difference in potential outcomes when data is generated from our model (Example 1) or closely generated from our model (Example 2). By contrast, IPW, g-estimation, and fitting our model without the latent variable provided significantly worse estimates in both examples to the point of drawing the wrong conclusion. That is, these alternative methods predict an admission carries a higher risk than a discharge, when in fact an admission carries a lower risk than a discharge. These methods did not adequately adjust for the risk of discharging associated with high-needs individual. A similar discrepancy between our method and these alternative methods was found in our case study. While our method predicts that an admission carries a lower risk than a discharge, IPW and g-estimation predict that an admission carries a higher risk than a discharge. Hence, our method is providing different estimates than other causal inferences.

There are several aspects about our method that should be carefully considered. One consideration is that our causal approach depends on a latent-variable model. Model dependence is a common concern with all causal inference approaches because estimates generally depend on model choice. For example, we expect estimates of mean difference in potential outcomes to improve with better models of the admission process. Indeed, when data is simulated from our model (Example 1) or closely simulated from our model (Example 2), we find that our approach yields significantly better estimates of this risk difference than IPW or g-estimation approaches. Model-dependence, however, is not a unique concern to our approach. All causal inferences require a conceptual model or hypothesis of the problem, at the very least, to specify to what extent certain variables could influence both treatment assignments and outcomes. For the classic question of whether smoking causes lung cancer, one might hypothesize that poor habits in general (eg, excessive drinking) could be associated with increased smoking and lung cancer. Causal methods can be made robust to violations in the mathematical model ( $\mathrm{cf}$ the work of Kennedy et $\mathrm{al}^{49}$ ). However, the conceptual model can rarely be validated. ${ }^{22}$ In other words, the model should be chosen carefully to recover accurate estimates from causal inference approaches. We thus strove to present a model that best reflects the actual admission decision process.

Even if less robust to model violations, a latent-variable approach could still provide more useful information in certain contexts compared to other causal information approaches. In our case study, for example, we estimated that admitting an individual with lower health needs increased the risk of an ED revisit by $3.0 \%$ and readmission by $7.6 \%$ but discharging an individual with higher health needs increased the risk of an ED revisit by $79.9 \%$ and readmission by $84.5 \%$. In other words, admitting a patient leads to worse outcomes only for lower needs individuals, suggesting efforts to decrease admission rates could be welcome, provided it did not impact higher needs individuals. In contrast, g-estimation and IPW estimated that admitting a general individual increased the risk of an ED revisit by about $2 \%$ and readmission by about $9 \%$. This result would also support efforts to decrease admission rates but would not bring the caveat that discharging certain individuals could be disastrous.

Researchers in the clinical community have employed different empirical approaches to similar questions. In the ED, for example, Stowell et $\mathrm{al}^{50}$ use a matched pair cluster study to compare quality of care (ie, length of stay, mortality, hospital readmissions, and rate of transfer to the ICU) for patients outlying in inappropriate wards after admission because of lack of vacant beds in appropriate specialty wards to the care given to nonoutlying patients. Empirical techniques have similarly been used to assess outcomes from the care process in the ICU (cf the works of Suter et al,,$^{51}$ Azoulay et al, ${ }^{52}$ and Simchen et $\mathrm{al}^{53}$ ). Related to the present study are those assessing the impact of length of stay on patient outcomes (cf the works of Bueno et al,${ }^{54}$ Williams et al,${ }^{55}$ Nichols et al,${ }^{56}$ Reynolds et al,${ }^{57}$ and Kaboli et al ${ }^{58}$ ). 
We remark that transfer decisions, such as ED admission decisions, are routine in hospital units (cf the work of Odetola et $\mathrm{al}^{59}$ ). We contend that our approach is sufficiently broad that it could capture many of these transfer decisions, but specific enough to provide insight to the transfer decision of interest. In addition, and as alluded to, we assume that both observations beyond an initial observation and the number of decisions until the final decision to transfer/discharge are completely missing from the data. This assumption ensures that observational data can be analyzed with both minimal restrictions: only an initial observation, final decisions, length of stay, and demographic information are needed; and, in general, populations.

However, our approach is not without limitations. First is that admission decisions and, in general, transfer decisions between hospital units may or may not depend on "operational factors" such as congestion, patient home environment, and hospital size (cf related works ${ }^{10,60-66}$ ), which need to be accounted for when assessing the causal impact of admission and transfer decisions on patient outcomes. Second is that we used a threshold regression model of the disposition decision. Estimating causal effects using an alternative model of the admission decision and comparing with our model is of clear interest. One alternative model is described in Section 2.4: use a more conventional survival model such as a Weibull regression model for treatment time and an another model for the admission decision conditional on the treatment time. This approach would require 2 additional parameters to capture main effects (characteristics, acuity, and health state in both models and treatment time or admission decision in the second model). More parameters would be needed for interaction terms to reproduce the observation in the data, whereby a mismatch between acuity and admission decision leads to longer treatment times. Third is that we apply our approach to a general ED population after restricting to those patients with ESI index 2 and 3 and excluding patients admitted to the ICU. EHR, however, may contain additional patient-specific information, such as chief complaint, that can be leveraged at baseline. Fourth, EHR may also contain intermediate information (eg, vitals, lab tests) that could strengthen causal relationships. However, mapping this information onto observations in the admission decision process is expected to require additional models and assumptions that are specific to a condition and/or hospital. Fifth, we chose a specific form of our parametric model of potential outcomes in which only health needs have a direct effect. However, one may want to include patient characteristics, acuity, and treatment time in this model if these variables are believed to have a direct effect on potential outcomes even when controlling for health state.

\section{ACKNOWLEDGEMENTS}

Cochran's contributions were supported by grant K01MH112876 from the National Institute of Mental Health. Rathouz' effort on this project was supported by NIH Grant R01HL094786-05A1.

\section{CONFLICT OF INTEREST}

The authors declare no potential conflict of interests.

\section{ORCID}

Gabriel Zayas-Cabán (10) https://orcid.org/0000-0002-3442-2768

\section{REFERENCES}

1. Schuur JD, Venkatesh AK. The growing role of emergency departments in hospital admissions. N Engl J Med. 2012;367(5):391-393.

2. Kocher KE, Dimick JB, Nallamothu BK. Changes in the source of unscheduled hospitalizations in the united states. Medical Care. 2013;51(8):689-698.

3. Medicare Centers, Services Medicaid. Medicare benefit policy manual, chapter 6: hospital services covered under part B. https://www. cms.gov/Regulations-and-Guidance/Guidance/Manuals/Downloads/bp102c06.pdf. 2011. Accessed November $29,2016$.

4. Damiani G, Pinnarelli L, Sommella L, Vena V, Magrini P, Ricciardi W. The short stay unit as a new option for hospitals: a review of the scientific literature. Med Sci Monit. 2011;17(6):SR15-SR19.

5. Noel-Miller C, Lind K. Is observation status substituting for hospital readmission? Health Affairs Blog. 2015.

6. Zuckerman RB, Sheingold SH, Orav EJ, Ruhter J, Epstein AM. Readmissions, observation, and the hospital readmissions reduction program. N Engl J Med. 2016;374(16):1543-1551. 
7. Sabbatini AK, Nallamothu BK, Kocher KE. Reducing variation in hospital admissions from the emergency department for low-mortality conditions may produce savings. Health Affairs. 2014;33(9):1655-1663.

8. Kim S-H, Chan CW, Olivares M, Escobar G. ICU admission control: an empirical study of capacity allocation and its implication for patient outcomes. Management Science. 2014;61(1):19-38.

9. Chan CW, Green LV, Lu L, Escobar G. The Role of a Step-Down Unit in Improving Patient Outcomes. Working paper. New York, NY: Columbia Business School; 2014.

10. Kim SH, Chan CW, Olivares M, Escobar GJ. Association among ICU congestion, ICU admission decision, and patient outcomes. Crit Care Med. 2016;44(10):1814-1821.

11. Bartel AP, Chan CW, Kim S-H(Hailey). Should Hospitals Keep Their Patients Longer? The Role of Inpatient Care in Reducing Post-Discharge Mortality. Working paper 20499. Cambridge, MA: National Bureau of Economic Research; 2014.

12. Kuntz L, Scholtes S, Sülz S. Separate \& Concentrate: Accounting for Process Uncertainty in the Design of Regional Hospital Systems. Working paper. Cambridge, UK: Cambridge University; 2016.

13. Hernán MA, Robins JM. Instruments for causal inference: an epidemiologist's dream? Epidemiology. 2006;17(4):360-372.

14. Deaton A. Instruments, randomization, and learning about development. J Econ Lit. 2010;48(2):424-455.

15. Imbens GW. Better late than nothing: some comments on Deaton (2009) and Heckman and Urzua (2009). J Econ Lit. 2010;48(2):399-423.

16. Rosenbaum PR, Rubin DB. The central role of the propensity score in observational studies for causal effects. Biometrika. 1983;70(1): 41-55.

17. Seaman SR, White IR. Review of inverse probability weighting for dealing with missing data. Stat Methods Med Res. 2013;22(3):278-295.

18. Kim J-O, Ferree GD Jr. Standardization in causal analysis. Sociol Methods Res. 1981;10(2):187-210.

19. Witteman JCM, D'Agostino RB, Stijnen T, et al. G-estimation of causal effects: isolated systolic hypertension and cardiovascular death in the framingham heart study. Am J Epidemiol. 1998;148(4):390-401.

20. Rosenbaum PR. Observational Studies. New York, NY:Springer; 2002.

21. Pearl J. Causality. Cambridge, UK: Cambridge University Press; 2009.

22. Hernan MA, Robins JM. Causal Inference. Boca Raton, FL: CRC Press; 2010.

23. Morgan SL, Winship C. Counterfactuals and Causal Inference. Cambridge, UK: Cambridge University Press; 2014.

24. Bentler PM. Multivariate analysis with latent variables: causal modeling. Annu Rev Psychol. 1980;31(1):419-456.

25. Berkane M. Latent Variable Modeling and Applications to Causality; vol. 120. New York, NY: Springer Science \& Business Media; 2012.

26. Pearl J. On the testability of causal models with latent and instrumental variables. In: Proceedings of the 11th Conference on Uncertainty in Artificial Intelligence (UAI'95); 1995; Quebec, Canada.

27. Pearl J, Robins J. Probabilistic evaluation of sequential plans from causal models with hidden variables. In: Proceedings of the 11th Annual Conference on Uncertainty in Artificial Intelligence (UAI'95); 1995; Quebec, Canada.

28. Heckman JJ, Vytlacil EJ. Local instrumental variables and latent variable models for identifying and bounding treatment effects. Proc Natl Acad Sci. 1999;96(8):4730-4734.

29. Gadbury GL, Supapakorn T, Coffey CS, Keith SW, Allison DB. Application of potential outcomes to an intentional weight loss latent variable problem. Stat Interface. 2008;1(1):87.

30. Bartolucci F. On the conditional logistic estimator in two-arm experimental studies with non-compliance and before-after binary outcomes. Statist Med. 2010;29(13):1411-1429.

31. Bartolucci F, Farcomeni A. Causal inference in paired two-arm experimental studies under noncompliance with application to prognosis of myocardial infarction. Statist Med. 2013;32(25):4348-4366.

32. Louizos C, Shalit U, Mooij JM, Sontag D, Zemel R, Welling M. Causal effect inference with deep latent-variable models. In: Proceedings of the Advances in Neural Information Processing Systems 30 (NIPS 2017); 2017; Long Beach, CA.

33. Krajbich I, Rangel A. Multialternative drift-diffusion model predicts the relationship between visual fixations and choice in value-based decisions. Proc Natl Acad Sci. 2011;108(33):13852-13857.

34. Lee MLT, Whitmore GA. Threshold regression for survival analysis: modeling event times by a stochastic process reaching a boundary. Statistical Science. 2006;21(4):501-513.

35. Navarro DJ, Fuss IG. Fast and accurate calculations for first-passage times in wiener diffusion models. J Math Psychol. 2009;53(4): 222-230.

36. Saunders NR, To T, Parkin PC, Guttmann A. Emergency department revisits by urban immigrant children in canada: a population-based cohort study. J Pediatr. 2016;170:218-226.

37. Kocher RP, Adashi EY. Hospital readmissions and the affordable care act: paying for coordinated quality care. JAMA. 2011;306(16):1794-1795.

38. Knott M, Bartholomew DJ. Latent Variable Models and Factor Analysis. London, UK: Edward Arnold; 1999.

39. Ratcliff R, McKoon G. The diffusion decision model: theory and data for two-choice decision tasks. Neural Comput. 2008;20(4):873-922.

40. Whitmore G. The inverse Gaussian distribution as a model of hospital stay. Health Serv Res. 1975;10(3):297-302.

41. Eaton WW, Whitmore G. Length of stay as a stochastic process: a general approach and application to hospitalization for schizophrenia. J Math Sociol. 1977;5(2):273-292.

42. Ting Lee ML, DeGruttola V, Schoenfeld D. A model for markers and latent health status. J R Stat Soc Ser B Stat Methodol. 2000;62(4):747-762. 
43. Dempster AP, Laird NM, Rubin DB. Maximum likelihood from incomplete data via the EM algorithm. J R Stat Soc Ser B Methodol. 1977;39:1-22.

44. Oakes D. Direct calculation of the information matrix via the EM. J R Stat Soc Ser B Stat Methodol. 1999;61(2):479-482.

45. Bolck A, Croon M, Hagenaars J. Estimating latent structure models with categorical variables: one-step versus three-step estimators. Political Analysis. 2004;12(1):3-27.

46. Dziak JJ, Bray BC, Zhang J, Zhang M, Lanza ST. Comparing the performance of improved classify-analyze approaches for distal outcomes in latent profile analysis. Methodology. 2016;12(4):107-116.

47. Chen J, Shao J. Nearest neighbor imputation for survey data. J Off Stat. 2000;16(2):113-131.

48. Rubin DB. Causal inference using potential outcomes: design, modeling, decisions. J Am Stat Assoc. 2005;100(469):322-331.

49. Kennedy EH, Ma Z, McHugh MD, Small DS. Non-parametric methods for doubly robust estimation of continuous treatment effects. J R Stat Soc Ser B Stat Methodol. 2017;79(4):1229-1245.

50. Stowell A, Claret PG, Sebbane M, et al. Hospital out-lying through lack of beds and its impact on care and patient outcome. Scand J Trauma Resusc Emerg Med. 2013;21(1):17.

51. Suter P, Armaganidis A, Beaufils F, et al. Predicting outcome in ICU patients. Intensive Care Med. 1994;20(5):390-397.

52. Azoulay Élie, Pochard F, Chevret S, et al. Compliance with triage to intensive care recommendations. Crit Care Med. 2001;29(11):2132-2136.

53. Simchen E, Sprung CL, Galai N, et al. Survival of critically ill patients hospitalized in and out of intensive care units under paucity of intensive care unit beds. Crit Care Med. 2004;32(8):1654-1661.

54. Bueno H, Ross JS, Wang Y, et al. Trends in length of stay and short-term outcomes among medicare patients hospitalized for heart failure, 1993-2006. JAMA. 2010;303(21):2141-2147.

55. Williams TA, Ho KM, Dobb GJ, Finn JC, Knuiman M, Webb SAR. Effect of length of stay in intensive care unit on hospital and long-term mortality of critically ill adult patients. Br J Anaesth. 2010;104(4):459-464.

56. Nichols GA, Reynolds K, Butler MG, Kimes TM, Chan WW. Association of acute heart failure length of stay with subsequent re-hospitalization and all-cause mortality among patients with preserved vs. reduced ejection fraction. Circulation. 2014;130:A11307.

57. Reynolds K, Butler MG, Kimes TM, Rosales AG, Chan W, Nichols GA. Relation of acute heart failure hospital length of stay to subsequent readmission and all-cause mortality. Am J Cardiol. 2015;116(3):400-405.

58. Kaboli PJ, Go JT, Hockenberry J, et al. Associations between reduced hospital length of stay and 30-day readmission rate and mortality: 14-year experience in 129 veterans affairs hospitals. Ann Intern Med. 2012;157(12):837-845.

59. Odetola FO, Bruski L, Zayas-Caban G, Lavieri M. An innovative framework to improve efficiency of interhospital transfer of children in respiratory failure. Ann Am Thorac Soc. 2016;13(5):671-677.

60. Kc DS, Terwiesch C. An econometric analysis of patient flows in the cardiac intensive care unit. Manuf Serv Oper Manag. 2012;14(1):50-65.

61. Abir M, Goldstick JE, Malsberger R, et al. Evaluating the impact of emergency department crowding on disposition patterns and outcomes of discharged patients. Int J Emerg Med. 2019;12(1):4.

62. Calder LA, Forster AJ, Stiell IG, et al. Mapping out the emergency department disposition decision for high-acuity patients. Ann Emerg Med. 2012;60(5):567-576.

63. Gorski JK, Batt RJ, Otles E, Shah MN, Hamedani AG, Patterson BW. The impact of emergency department census on the decision to admit. Acad Emerg Med. 2017;24(1):13-21.

64. Nugus P, Carroll K, Hewett DG, Short A, Forero R, Braithwaite J. Integrated care in the emergency department: a complex adaptive systems perspective. Soc Sci Med. 2010;71(11):1997-2004.

65. Florin TA, French B, Zorc JJ, Alpern ER, Shah SS. Variation in emergency department diagnostic testing and disposition outcomes in pneumonia. Pediatrics. 2013;132(2):237-244.

66. Studnicki J, Platonova EA, Fisher JW. Hospital-level variation in the percentage of admissions originating in the emergency department. Am J Emerg Med. 2012;30(8):1441-1446.

How to cite this article: Cochran AL, Rathouz PJ, Kocher KE, Zayas-Cabán G. A latent variable approach to potential outcomes for emergency department admission decisions. Statistics in Medicine. 2019;38:3911-3935. https://doi.org/10.1002/sim.8210 


\section{APPENDIX A}

\section{TABLE OF PARAMETER ESTIMATES FOR SIMULATION}

TABLE A1 Performance of parameter estimates when the model is correct (Example 1) in terms of bias, mean square error (MSE), and percent coverage of true parameters for $95 \%$ confidence intervals. True parameter values are also reported. Variable $X_{2}$ was uniformly distributed between $-1 / 2$ and $1 / 2$

\begin{tabular}{|c|c|c|c|c|c|c|c|c|}
\hline & & & & $\mathbf{N}=1000$ & & & $\mathbf{N}=\mathbf{1 0 0}$ & \\
\hline & & Value & Bias & MSE & Coverage & Bias & MSE & Coverage \\
\hline $\operatorname{logit} \alpha(X)$ & Intercept & -0.85 & -0.0112 & 0.0743 & 0.98 & -0.0005 & 0.0056 & 0.94 \\
\hline & $X_{1}=1$ & -0.25 & 0.0121 & 0.0555 & 0.96 & 0.0008 & 0.0049 & 0.92 \\
\hline & $X_{2}$ & & 0.0441 & 0.2444 & 0.94 & 0.0133 & 0.018 & 0.94 \\
\hline $\operatorname{logit} \beta(X, H)$ & $H=0$ & -0.41 & -0.0342 & 0.0620 & 1.00 & -0.0049 & 0.0016 & 0.92 \\
\hline & $H=1$ & 1.39 & 1.5758 & 44.4350 & 0.94 & 0.0128 & 0.0158 & 0.94 \\
\hline & $X_{1}=1$ & 0 & -0.0056 & 0.0256 & 1.00 & 0.0043 & 0.0023 & 0.94 \\
\hline & $X_{2}$ & 0 & -0.0628 & 0.1684 & 0.98 & -0.0003 & 0.0069 & 0.92 \\
\hline $\log |d|(H)$ & $H=0$ & 0.41 & 0.0059 & 0.0053 & 0.94 & 0.0043 & 0.0005 & 0.94 \\
\hline & $H=1$ & 0.69 & -0.0048 & 0.0061 & 0.96 & 0.0011 & 0.0006 & 0.90 \\
\hline $\operatorname{logit} c(X, Z)$ & $Z=0$ & -0.41 & -0.0051 & 0.0053 & 0.98 & 0.0001 & 0.0006 & 0.90 \\
\hline & $Z=1$ & 0 & 0.0040 & 0.0190 & 0.98 & -0.0007 & 0.0019 & 0.92 \\
\hline & $X_{1}=1$ & 0 & -0.0146 & 0.0270 & 0.94 & -0.007 & 0.0025 & 0.98 \\
\hline & $X_{2}$ & 0 & -0.0606 & 0.1045 & 0.86 & -0.0161 & 0.0032 & 0.96 \\
\hline $\log b(X)$ & Intercept & 0 & 0.0101 & 0.0013 & 0.90 & 0.006 & 0.0002 & 0.92 \\
\hline & $X_{1}=1$ & 0 & 0.0006 & 0.0013 & 0.94 & 0.001 & 0.0001 & 0.90 \\
\hline & $X_{2}$ & 0 & -0.0020 & 0.0040 & 0.94 & -0.0001 & 0.0004 & 0.96 \\
\hline$\mu_{1}$ & Intercept & 0.1 & 0.0063 & 0.0005 & 0.94 & 0.0006 & 0.0001 & 0.90 \\
\hline$\mu_{2}$ & Intercept & 0.9 & -0.1152 & 0.0801 & 0.94 & -0.022 & 0.013 & 0.88 \\
\hline$\mu_{3}$ & Intercept & 0.2 & 0.0073 & 0.0177 & 0.96 & 0.0004 & 0.001 & 0.96 \\
\hline$\mu_{4}$ & Intercept & 0.2 & -0.0009 & 0.0025 & 0.90 & -0.0004 & 0.0002 & 0.98 \\
\hline
\end{tabular}

\section{APPENDIX B}

\section{JOINT DENSITY APPROXIMATION FOR ADMISSION DECISION AND TREATMENT TIME}

In the main text, we introduced a structural model of the joint distribution of the admission decision $A$ and treatment time $T$ conditional on patient characteristics $X$, health needs $H$, and initial observation $Z$. The density function for $(A, T)=(a, t)$ conditional on $X, Z, H$ was expressed in the form $g(a, t \mid b(X), c(X, Z), d(H))$ with $b(X), c(X, Z) b(X), d(H) b(X)$ representing respectively an upper boundary, starting point, and drift rate. Dropping the explicit dependence on $X, Z, H$, the density function $g(a, t \mid b, c, d)$ can be expressed exactly ${ }^{35}$ as either

$$
g(a, t \mid b, c, d)=\exp \left(-(d b) b c-\frac{(d b)^{2} t}{2}\right) \frac{\pi}{b^{2}} \sum_{k=1}^{\infty} k \exp \left(-\frac{k^{2} \pi^{2} t}{2 b^{2}}\right) \sin (k \pi c)
$$

or

$$
g(a, t \mid b, c, d)=\exp \left(-(d b) b c-\frac{(d b)^{2} t}{2}\right) \frac{1}{b^{2}} \frac{b^{3}}{\sqrt{2 \pi t^{3}}} \sum_{k=-\infty}^{\infty}(c+2 k) \exp \left(-\frac{(c+2 k)^{2} b^{2}}{2 t}\right) .
$$

We can thus approximate $g(a, t \mid b, c, d)$ by truncating either series. However, the number of terms needed for an accurate approximation depends on the value of $t$. When $t$ is large, $g$ can be accurately approximated with relatively few terms from the second approximation. When $t$ is small, $g$ can be accurately approximated with relatively few terms from the second approximation. We found that $g$ could be approximated accurately using the first approximation truncated to 10 terms (from $k=1$ to $k=10$ ) when $t / b^{2} \geq 1 / 10$ and using the second approximation truncated to 21 terms (from $k=-10$ to $k=10)$ when $t / b^{2}<1 / 10$. 


\section{APPENDIX C}

\section{ALTERNATIVE APPROACH TO PARAMETER ESTIMATION}

We present an alternative approach to estimate unknown parameters. There are three reasons to consider another approach, since MLE yields an asymptotically efficient estimator provided model distributions are correctly specified. First, one may estimate the distribution of latent variables prior to considering its effect on outcomes and then later decide to use these estimate to estimate outcomes. Second, one may not specify a distribution for outcomes. Third, the model may be incorrect, which raises several practical issues, depending on which part of the model is incorrect. Latent variables are hypothetical constructs for the purpose of understanding admission decisions; generally, there are no methods for directly measuring them. ${ }^{38}$ Therefore, one may overlook incorrect models for latent variables. An incorrect model is more worrisome when estimating outcomes, since changes in policy will stem from knowledge of how admission decisions causally impact outcomes. An incorrect model of outcomes might then influence interpretation of latent variables, if we try to simultaneously estimate latent states and outcomes. We thus propose an alternative approach that disentangles the estimation of the latent variable from the estimation of outcomes. A similar idea of separating estimating latent variables and effects of latent states on outcomes in several steps can be found in the work of Bolck et al. ${ }^{45}$ In the work of Dziak et al ${ }^{46}$ the authors show numerically that, in certain cases, the approach in the work of Bolck et al ${ }^{45}$ can be more robust to model violations.

To describe the estimation approaches, let $\theta$ be a set of model parameters and let $\Theta$ be the feasible set for $\theta$. We first assume that we can decompose $\theta$ and $\Theta$ as $\left(\theta_{1}, \theta_{2}\right)$ and $\Theta_{1} \otimes \Theta_{2}$ so that $\theta_{1}$ fully specifies the part of the model that does not involve outcomes $Y$, ie,

$$
f(z, a, t \mid x ; \theta)=f\left(z, a, t \mid x ; \theta_{1}\right) .
$$

Second, we assume that both the $\log$-likelihood function $\log f(z, a, t, y \mid x ; \theta)$ of data $(z, a, t, y)$ and the log-likelihood function $\log f\left(z, a, t \mid x ; \theta_{1}\right)$ of the data $(z, a, t)$ with outcomes excluded satisfy the typical regularity conditions needed for MLE, eg, identifiability and continuity.

An MLE approach would search for $\left(\theta_{1}, \theta_{2}\right) \in \Theta_{1} \otimes \Theta_{2}$ that maximizes

$$
\sum_{n} \log f\left(z_{n}, a_{n}, t_{n}, y_{n} \mid x_{n} ; \theta_{1}, \theta_{2}\right)=\sum_{n} \log f\left(y_{n} \mid x_{n}, z_{n}, a_{n}, t_{n} ; \theta_{1}, \theta_{2}\right)+\sum_{n} \log f\left(z_{n}, a_{n}, t_{n} \mid x_{n} ; \theta_{1}\right) .
$$

Under standard regularity conditions and provided the model is correctly specified, we know that the maximum likelihood estimator is a consistent and asymptotically-efficient estimator of the true value of $\left(\theta_{1}, \theta_{2}\right)$. These properties can be derived from the theory of estimating functions by noting that the maximum likelihood estimator satisfies

$$
\sum_{n}\left[\begin{array}{c}
\nabla_{\theta_{1}} \log f\left(y_{n} \mid x_{n}, z_{n}, a_{n}, t_{n} ; \theta_{1}, \theta_{2}\right)+\nabla_{\theta_{1}} \log f\left(z_{n}, a_{n}, t_{n} \mid x_{n}, \theta_{1}\right) \\
\nabla_{\theta_{2}} \log f\left(y_{n} \mid z_{n}, a_{n}, t_{n} ; \theta_{1}, \theta_{2}\right)
\end{array}\right]=0
$$

where the left-hand side is an estimating function with expectation 0.

Our alternative approach is a two-step approach, whereby MLE is performed once and then generalized estimating equation approach is used next. Step 1 ignores information on outcomes $Y$ and searches for $\theta_{1} \in \Theta_{1}$ that maximizes $\sum_{n} \log f\left(z_{n}, a_{n}, t_{n} \mid x_{n} ; \theta_{1}\right)$. Assumed regularity conditions ensure that the maximum likelihood estimator is a consistent and asymptotically-efficient (in the sense when $Y$ is ignored) estimator of the true value of $\theta_{1}$. These properties also follow from the MLE estimator satisfying

$$
\sum_{n} \nabla_{\theta_{1}} \log f\left(z_{n}, a_{n}, t_{n} \mid x_{n}, \theta_{1}\right)=0,
$$

where the left-hand side is an estimating equation of $\theta_{1}$ with mean zero. If $\hat{\theta}_{1}$ is the resulting estimator, Step 2 then estimates $\hat{\theta}_{2}$ using a generalized estimating equation. Together, the two steps amount to solving

$$
\sum_{n}\left[\begin{array}{c}
\nabla_{\theta_{1}} \log f\left(z_{n}, a_{n}, t_{n} \mid x_{n}, \theta_{1}\right) \\
\frac{\partial \mu^{T}}{\partial \theta_{1}} V_{n}(\mu)^{-1}\left(y_{n}-\mu\left(x_{n}, z_{n}, a_{n}, t_{n} ; \theta_{1}, \theta_{2}\right)\right)
\end{array}\right]=0,
$$

where functions $\mu$ and $V_{n}(\mu)$ are defined to respectively approximate the mean and variance of $Y$ given $X, Z, A, T$ and parameters $\theta_{1}, \theta_{2}$. Only $\mu$ needs to be correctly specified for the left-hand side of $(\mathrm{C} 1)$ to be an estimating function of $\theta_{1}, \theta_{2}$ with mean zero. The two-step MLE/GEE approach will thus also yield a consistent estimator of $\left(\theta_{1}, \theta_{2}\right)$. 


\section{C.1 Handling the latent variable}

Finally, we turn to discuss how to handle latent-variables $H$. It is easiest to define the joint distribution of all the random variables including $H$. In such a case, $f\left(y \mid x, z, a, t ; \theta_{1}, \theta_{2}\right)$ and $f\left(z, a, t \mid x, \theta_{1}\right)$ are not explicitly available, and so, we use expectation-maximum algorithm to perform MLE. ${ }^{43}$ We also note that

$$
\begin{aligned}
\mathbb{E}\left(y \mid x, z, a, t, \theta_{1}, \theta_{2}\right) & =\sum_{h} \mathbb{E}\left(y \mid x, z, a, t, h, \theta_{1}, \theta_{2}\right) \mathbb{P}\left(H=h \mid x, z, a, t, \theta_{1}, \theta_{2}\right) \\
& =\sum_{h} \mathbb{E}\left(y \mid x, z, a, t, h, \theta_{1}, \theta_{2}\right) \mathbb{P}\left(H=h \mid x, z, a, t, \theta_{1}\right) \\
& =\sum_{h} \mathbb{E}\left(y \mid x, z, a, t, h, \theta_{2}\right) \mathbb{P}\left(H=h \mid x, z, a, t, \theta_{1}\right) \\
& =\sum_{h} \mathbb{E}\left(y^{a} \mid x, z, t, h, \theta_{2}\right) \mathbb{P}\left(H=h \mid x, z, a, t, \theta_{1}\right),
\end{aligned}
$$

where we first drop $\theta_{1}$ from $\mathbb{E}\left(y \mid x, z, a, t, h, \theta_{1}, \theta_{2}\right)$ since we conditioned on $H=h$ along with the other variables; then drop $\theta_{2}$ in $\mathbb{P}\left(H=h \mid x, v, z, a, t, \theta_{1}\right)$ because of how parameters $\theta$ were decomposed; and, finally, use Assumption 1 to relate estimation of the distribution of outcomes $Y$ with estimation of the distribution of potential outcomes $Y^{a}$. These expressions mean that estimation of $\theta_{2}$ is linked to the estimation of $\theta_{1}$ only through $\mathbb{P}\left(H=h \mid x, z, a, t, \theta_{1}\right)$. Therefore, even if the latent-variable model or $\theta_{1}$ is incorrect, we can still estimate $\theta_{2}$ correctly and thereby make correct conclusions about potential outcomes provided the model for outcomes and $\mathbb{P}\left(H=h \mid x, z, a, t, \theta_{1}\right)$ are correct. These expressions also motivate choosing $\mu\left(x, z, a, t ; \theta_{1}, \theta_{2}\right)$ in Step 2 in the two-step MLE/GEE approach to be of the form

$$
\mu\left(x, z, a, t ; \theta_{1}, \theta_{2}\right):=\sum_{h} v_{h, a}\left(x, z, t, \theta_{2}\right) \mathbb{P}\left(H=h \mid x, z, a, t, \theta_{1}\right),
$$

for functions $v_{h, a}\left(x, z, t, \theta_{2}\right)$ that model $\mathbb{E}\left[Y^{a} \mid x, z, t, h, \theta_{2}\right]$.

TABLE C1 Bias and mean square error (MSE) for two-step parameter estimates. True values of parameters are also reported (Example 1)

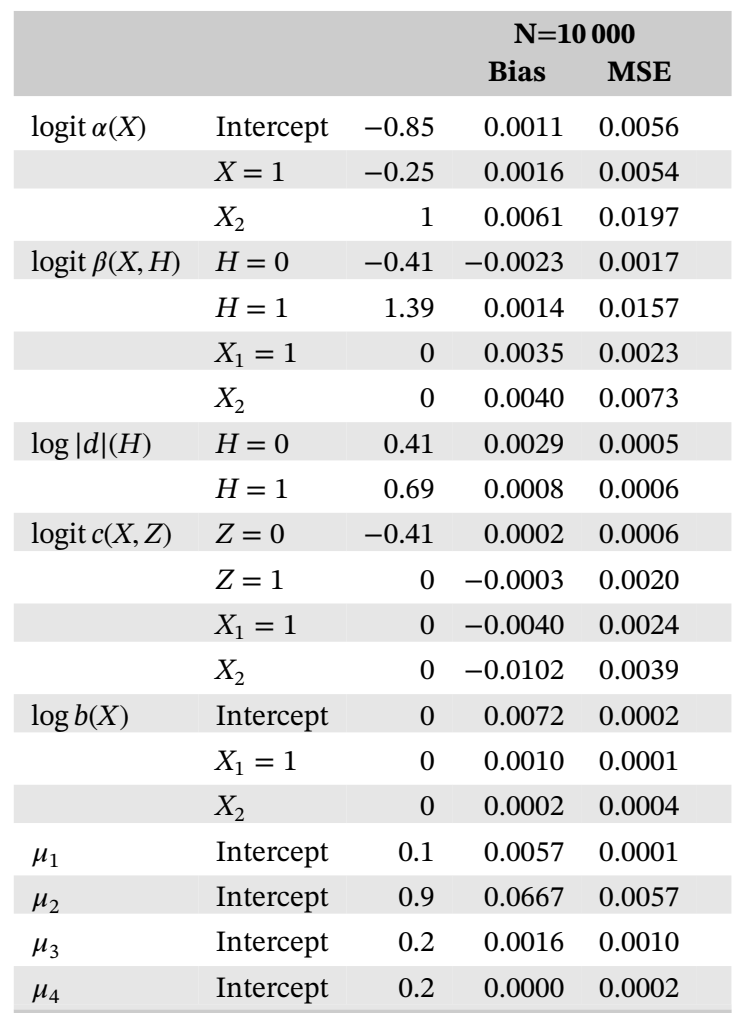


TABLE C2 Bias and mean square error (MSE) for two-step parameter estimates. True values of parameters are also reported (Example 2)

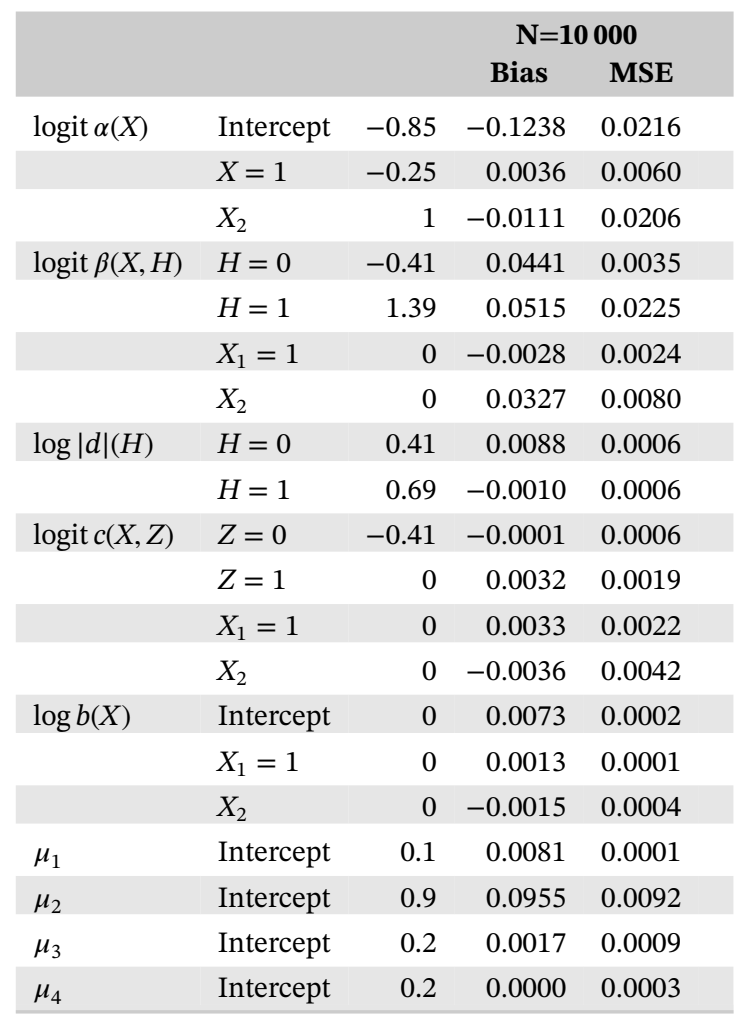

\section{C.2 Simulation results for a two-step approach}

We applied our estimation approach to data from the same two simulation examples in the main text with $N=10000$. Results can be found in Tables C1 and C2. In both examples, our two-step approach has similar performance in terms of bias and MSE to the one-step approach for nearly all the estimated parameters. In Example 1, the two-step estimate of $\mu_{2}$ is arguably the worst when compared to the one-step estimate of $\mu_{2}$, with bias equal to 0.0667 compared to -0.022 . However, in Example 2, the two-step estimate of $\mu_{2}$ has nearly the same bias as the one-step estimate of $\mu_{2}$, with bias equal to 0.0955 compared to 0.0958 .

\section{APPENDIX D}

\section{JUSTIFICATION OF PARAMETER CHOICES FOR SIMULATION}

Patient characteristics $X=\left(X_{1}, X_{2}\right)$ were defined with $X_{1}$ describing a binary characteristic such as gender and $X_{2}$ describing a numerical variable such as age. We chose an even $50 \%$ of simulated individuals to have $X_{1}=1$ and chose $X_{2}$ to be a uniformly random variable between $-1 / 2$ and $1 / 2$, where, for reference, about $45 \%$ of individuals visiting the ED are female and age are roughly uniformly-distributed from 18 to 75 years among ED visits. We chose parameters in a linear model of logit $\alpha(X)$ such that, on average, $30 \%$ of $X_{1}=0$ individuals have higher health needs $(H=1)$ and $25 \%$ of $X_{1}=1$ individuals have higher health needs $(H=1)$ to reflect the approximate $30 \%$ admission rates for men and $25 \%$ for women. We assumed that the linear coefficient for $X_{2}$ in logit $\alpha(X)$ was equal to 1 to reflect that older individuals are more likely to have higher health needs $(H=1)$.

For the remaining model components, we assume $X$ has no influence. We chose parameters for logit $\beta(X, H)$ such that $40 \%$ of individuals with lower health needs $(H=0)$ have a higher initial observation $(Z=1)$ compared to $80 \%$ of individuals with higher health needs $(H=1)$, which, when taken with the rate $\alpha(X)$ of latent health needs, reflects that about half of the individuals are assigned the higher acuity level (Acuity 2). We chose parameters for logit $c(X, Z)$ such that $c(X, Z)$ had a value of 0.5 for individuals with a higher initial observation $(Z=1)$ compared to 0.4 for individuals with a lower 
initial observation $(Z=0)$ to reflect that higher acuity patients are more likely to be admitted (about $42 \%$ of acuity 2 patients are admitted compared to $18 \%$ of acuity 3 patients). Because the drift rate term $d(H)$ and the boundary $b(X)$ are difficult to relate to the data and we can always rescale time in the simulation, we simply chose parameters to result in a value of 2 for $d(H)$ for higher health needs $(H=1)$ and -1.5 for lower health needs $(H=0)$ and a constant value of 1 for $b(X)$. Lastly, we assumed that $10 \%$ of lower needs patients have a poor outcome $Y=1$ when discharged $(A=0)$ compared to $20 \%$ when admitted $(A=1)$ and that $20 \%$ of higher needs patients have a worse outcome $Y=1$ when admitted $(A=1)$ compared to $90 \%$ when discharged $(A=0)$. These values were chosen to capture two potential trends: higher needs patients have worse outcomes than lower needs patients and a mismatch between needs and admission decisions leads to worse outcomes.

\section{APPENDIX E}

\section{TABLES OF PARAMETER ESTIMATES FOR CASE STUDY}

TABLE E1 Parameter estimates and 95\% confidence intervals (CIs) for admission decision model and causal effects of admissions on emergency department revisits. Parameters were expressed as a linear model transformed by a nonlinear link function. Estimates are reported for coefficients in the linear model. Variables were encoded such that male was zero and female was one; acuity 3 was zero and acuity 2 was one. Age was standardized and sex was centered to its mean

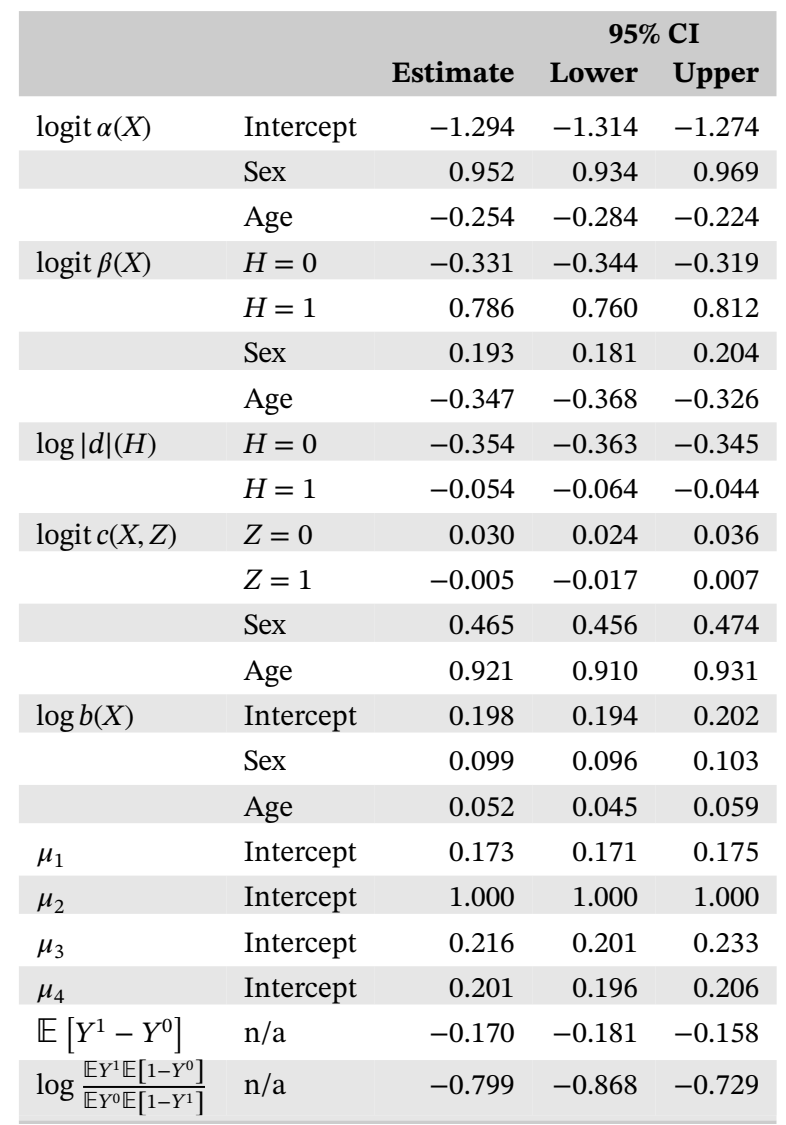


TABLE E2 Parameter estimates and 95\% confidence interval (CI) of causal effects of admission on emergency department readmission

\begin{tabular}{|llrrr} 
& & & \multicolumn{2}{c}{$95 \%$ CI } \\
& & Estimate & Lower & Upper \\
$\mu_{1}$ & Intercept & 0.030 & 0.029 & 0.031 \\
$\mu_{2}$ & Intercept & 0.999 & 0.995 & 1.000 \\
$\mu_{3}$ & Intercept & 0.106 & 0.091 & 0.123 \\
$\mu_{4}$ & Intercept & 0.154 & 0.149 & 0.158 \\
$\mathbb{E}\left[Y^{1}-Y^{0}\right]$ & n/a & -0.156 & -0.168 & -0.144 \\
$\log \frac{\mathbb{E} Y^{1} \mathbb{E}\left[1-Y^{0}\right]}{\mathbb{E} Y^{0} \mathbb{E}\left[1-Y^{1}\right]}$ & n/a & -0.883 & -0.998 & -0.767 \\
\hline
\end{tabular}

TABLE E3 Sensitivity of causal impact of admission on emergency department revisits to violations in Assumption 1, captured when $\psi_{0}$ and $\psi_{1}$ are not both 1. For each estimate, 95\% confidence intervals are provided

\begin{tabular}{|c|c|c|c|c|c|c|}
\hline$\psi_{0}$ & $\psi_{1}$ & $\mu_{1}$ & $\mu_{2}$ & $\mu_{3}$ & $\mu_{4}$ & $\mathbb{E}\left[\boldsymbol{Y}^{\mathbf{1}}-\boldsymbol{Y}^{\mathbf{0}}\right]$ \\
\hline 0.95 & 0.95 & $0.17(0.17,0.17)$ & $1.00(1.00,1.00)$ & $0.24(0.22,0.25)$ & $0.20(0.19,0.20)$ & $-0.15(-0.17,-0.14)$ \\
\hline 0.95 & 0.975 & $0.17(0.17,0.17)$ & $1.00(0.95,1.00)$ & $0.23(0.21,0.24)$ & $0.20(0.19,0.20)$ & $-0.16(-0.17,-0.15)$ \\
\hline 0.95 & 1 & $0.17(0.17,0.17)$ & $1.00(1.00,1.00)$ & $0.22(0.20,0.24)$ & $0.20(0.20,0.21)$ & $-0.17(-0.18,-0.16)$ \\
\hline 0.95 & 1.025 & $0.17(0.17,0.17)$ & $1.00(1.00,1.00)$ & $0.21(0.19,0.22)$ & $0.20(0.20,0.21)$ & $-0.18(-0.19,-0.16)$ \\
\hline 0.95 & 1.05 & $0.17(0.17,0.17)$ & $1.00(1.00,1.00)$ & $0.20(0.18,0.21)$ & $0.21(0.20,0.21)$ & $-0.18(-0.20,-0.17)$ \\
\hline 0.975 & 0.95 & $0.17(0.17,0.18)$ & $1.00(1.00,1.00)$ & $0.24(0.22,0.25)$ & $0.20(0.19,0.20)$ & $-0.15(-0.17,-0.14)$ \\
\hline 0.975 & 0.975 & $0.17(0.17,0.18)$ & $1.00(1.00,1.00)$ & $0.23(0.21,0.24)$ & $0.20(0.19,0.20)$ & $-0.16(-0.18,-0.15)$ \\
\hline 0.975 & 1 & $0.17(0.17,0.18)$ & $1.00(1.00,1.00)$ & $0.22(0.20,0.24)$ & $0.20(0.20,0.21)$ & $-0.17(-0.18,-0.16)$ \\
\hline 0.975 & 1.025 & $0.17(0.17,0.18)$ & $1.00(1.00,1.00)$ & $0.21(0.19,0.22)$ & $0.20(0.20,0.21)$ & $-0.18(-0.19,-0.16)$ \\
\hline 0.975 & 1.05 & $0.17(0.17,0.18)$ & & $0.20(0.18,0.21)$ & & $-0.18(-0.20,-0.17)$ \\
\hline 1 & & & & $0.24(0.22,0.25)$ & $0.20(0.19,0.20)$ & $-0.15(-0.17,-0.14)$ \\
\hline 1 & 0.975 & $0.17(0.17,0.18)$ & $1.00(1.00,1.00)$ & $0.23(0.21,0.25)$ & $0.20(0.19,0.20)$ & $-0.16(-0.17,-0.15)$ \\
\hline 1 & 1 & $0.17(0.17,0.18)$ & $1.00(1.00,1.00)$ & $0.22(0.20,0.23)$ & $0.20(0.20,0.21)$ & $-0.17(-0.18,-0.16)$ \\
\hline 1 & 1.025 & $0.17(0.17,0.18)$ & $1.00(1.00,1.00)$ & $0.21(0.19,0.22)$ & $0.20(0.20,0.21)$ & $-0.18(-0.19,-0.16)$ \\
\hline 1 & 1.05 & $0.17(0.17,0.18)$ & $1.00(1.00,1.00)$ & $0.19(0.18,0.21)$ & $0.21(0.20,0.21)$ & $-0.19(-0.20,-0.17)$ \\
\hline 1.025 & 0.95 & $0.17(0.17,0.18)$ & $1.00(0.99,1.00)$ & $0.24(0.22,0.26)$ & $0.20(0.19,0.20)$ & $-0.15(-0.17,-0.14)$ \\
\hline 1.025 & 0.975 & $0.17(0.17,0.18)$ & $1.00(0.99,1.00)$ & $0.23(0.21,0.25)$ & $0.20(0.19,0.20)$ & $-0.16(-0.18,-0.15)$ \\
\hline 1.025 & 1 & $0.17(0.17,0.18)$ & $1.00(1.00,1.00)$ & $0.22(0.20,0.23)$ & $0.20(0.20,0.21)$ & $-0.17(-0.18,-0.16)$ \\
\hline 1.025 & 1.025 & $0.17(0.17,0.18)$ & $1.00(0.99,1.00)$ & $0.21(0.19,0.22)$ & $0.20(0.20,0.21)$ & $-0.18(-0.19,-0.16)$ \\
\hline 1.025 & 1.05 & $0.17(0.17,0.18)$ & $1.00(1.00,1.00)$ & $0.19(0.18,0.21)$ & $0.21(0.20,0.21)$ & $-0.19(-0.20,-0.17)$ \\
\hline 1.05 & 0.95 & $0.17(0.17,0.18)$ & $0.99(0.99,0.99)$ & $0.24(0.22,0.26)$ & $0.20(0.19,0.20)$ & $-0.15(-0.17,-0.14)$ \\
\hline 1.05 & 0.975 & $0.17(0.17,0.18)$ & $0.99(0.99,0.99)$ & $0.23(0.21,0.25)$ & $0.20(0.19,0.20)$ & $-0.16(-0.17,-0.15)$ \\
\hline 1.05 & 1 & $0.17(0.17,0.18)$ & $0.99(0.99,0.99)$ & $0.22(0.20,0.24)$ & $0.20(0.20,0.21)$ & $-0.17(-0.18,-0.15)$ \\
\hline 1.05 & 1.025 & $0.17(0.17,0.18)$ & $0.99(0.99,0.99)$ & $0.21(0.19,0.22)$ & $0.20(0.20,0.21)$ & $-0.18(-0.19,-0.16)$ \\
\hline 1.05 & 1.05 & $0.17(0.17,0.18)$ & $0.99(0.99,0.99)$ & $0.19(0.18,0.21)$ & $0.21(0.20,0.21)$ & $-0.18(-0.20,-0.17)$ \\
\hline
\end{tabular}


TABLE E4 Sensitivity of causal impact of admission on emergency department readmissions to violations in Assumption 1, captured when $\psi_{0}$ and $\psi_{1}$ are not both 1. For each estimate, 95\% confidence intervals are provided

\begin{tabular}{|c|c|c|c|c|c|c|}
\hline$\psi_{0}$ & $\psi_{1}$ & $\mu_{1}$ & $\mu_{2}$ & $\mu_{3}$ & $\mu_{4}$ & $\mathbb{E}\left[Y^{1}-Y^{0}\right]$ \\
\hline 0.95 & 0.95 & $0.03(0.03,0.03)$ & $1.00(1.00,1.00)$ & $0.12(0.11,0.14)$ & $0.15(0.15,0.16)$ & $-0.14(-0.16,-0.13)$ \\
\hline 0.95 & 0.975 & $0.03(0.03,0.03)$ & $1.00(0.61,1.00)$ & $0.11(0.10,0.13)$ & $0.15(0.15,0.16)$ & $-0.15(-0.16,-0.14)$ \\
\hline 0.95 & 1 & $0.03(0.03,0.03)$ & $1.00(1.00,1.00)$ & $0.11(0.09,0.12)$ & $0.15(0.15,0.16)$ & $-0.16(-0.17,-0.14)$ \\
\hline 0.95 & 1.025 & $0.03(0.03,0.03)$ & $1.00(1.00,1.00)$ & $0.10(0.08,0.11)$ & $0.16(0.15,0.16)$ & $-0.16(-0.17,-0.15)$ \\
\hline 0.95 & 1.05 & $0.03(0.03,0.03)$ & $1.00(1.00,1.00)$ & $0.09(0.08,0.11)$ & $0.16(0.15,0.16)$ & $-0.17(-0.18,-0.16)$ \\
\hline 0.975 & 0.95 & $0.03(0.03,0.03)$ & $1.00(1.00,1.00)$ & $0.12(0.11,0.14)$ & $0.15(0.15,0.16)$ & $-0.14(-0.16,-0.13)$ \\
\hline 0.975 & 0.975 & $0.03(0.03,0.03)$ & $1.00(1.00,1.00)$ & $0.11(0.10,0.13)$ & $0.15(0.15,0.16)$ & $-0.15(-0.16,-0.14)$ \\
\hline 0.975 & 1 & $0.03(0.03,0.03)$ & $1.00(1.00,1.00)$ & $0.11(0.09,0.12)$ & $0.15(0.15,0.16)$ & $-0.16(-0.17,-0.14)$ \\
\hline 0.975 & 1.025 & $0.03(0.03,0.03)$ & $1.00(1.00,1.00)$ & $0.10(0.08,0.11)$ & $0.16(0.15,0.16)$ & $-0.16(-0.17,-0.15)$ \\
\hline 0.975 & 1.05 & $0.03(0.03,0.03)$ & $1.00(1.00,1.00)$ & $0.09(0.08,0.11)$ & $0.16(0.15,0.16)$ & $-0.17(-0.18,-0.16)$ \\
\hline 1 & 0.95 & $0.03(0.03,0.03)$ & $1.00(1.00,1.00)$ & $0.12(0.11,0.14)$ & $0.15(0.15,0.16)$ & $-0.14(-0.16,-0.13)$ \\
\hline 1 & & $0.03(0.03,0.03)$ & $1.00(1.00,1.00)$ & $0.11(0.10,0.13)$ & $0.15(0.15,0.16)$ & $-0.15(-0.16,-0.14)$ \\
\hline 1 & 1 & $0.03(0.03,0.03)$ & $1.00(1.00,1.00)$ & $0.11(0.09,0.12)$ & $0.15(0.15,0.16)$ & $-0.16(-0.17,-0.14)$ \\
\hline 1 & 1.025 & $0.03(0.03,0.03)$ & $1.00(1.00,1.00)$ & $0.10(0.08,0.11)$ & $0.16(0.15,0.16)$ & $-0.16(-0.17,-0.15)$ \\
\hline 1 & 1.05 & $0.03(0.03,0.03)$ & $1.00(1.00,1.00)$ & $0.09(0.08,0.11)$ & $0.16(0.15,0.16)$ & $-0.17(-0.18,-0.16)$ \\
\hline 1.025 & 0.95 & $0.03(0.03,0.03)$ & $1.00(1.00,1.00)$ & $0.12(0.11,0.14)$ & $0.15(0.15,0.16)$ & $-0.15(-0.16,-0.13)$ \\
\hline 1.025 & 0.975 & $0.03(0.03,0.03)$ & $1.00(1.00,1.00)$ & $0.11(0.10,0.13)$ & $0.15(0.15,0.16)$ & $-0.15(-0.16,-0.14)$ \\
\hline 1.025 & 1 & $0.03(0.03,0.03)$ & $1.00(1.00,1.00)$ & $0.11(0.09,0.12)$ & $0.15(0.15,0.16)$ & $-0.16(-0.17,-0.15)$ \\
\hline 1.025 & 1.025 & $0.03(0.03,0.03)$ & $1.00(1.00,1.00)$ & $0.10(0.09,0.11)$ & $0.16(0.15,0.16)$ & $-0.16(-0.17,-0.15)$ \\
\hline 1.025 & 1.05 & $0.03(0.03,0.03)$ & $1.00(1.00,1.00)$ & $0.09(0.08,0.11)$ & $0.16(0.15,0.16)$ & $-0.17(-0.18,-0.16)$ \\
\hline 1.05 & 0.95 & $0.03(0.03,0.03)$ & $1.00(1.00,1.00)$ & $0.12(0.11,0.14)$ & $0.15(0.15,0.16)$ & $-0.15(-0.16,-0.13)$ \\
\hline 1.05 & 0.975 & $0.03(0.03,0.03)$ & $1.00(1.00,1.00)$ & $0.11(0.10,0.13)$ & $0.15(0.15,0.16)$ & $-0.15(-0.16,-0.14)$ \\
\hline 1.05 & 1 & $0.03(0.03,0.03)$ & $1.00(1.00,1.00)$ & $0.11(0.09,0.12)$ & $0.15(0.15,0.16)$ & $-0.16(-0.17,-0.15)$ \\
\hline 1.05 & 1.025 & $0.03(0.03,0.03)$ & $1.00(1.00,1.00)$ & $0.10(0.08,0.11)$ & $0.16(0.15,0.16)$ & $-0.16(-0.17,-0.15)$ \\
\hline 1.05 & 1.05 & $0.03(0.03,0.03)$ & $1.00(1.00,1.00)$ & $0.09(0.08,0.11)$ & $0.16(0.15,0.16)$ & $-0.17(-0.18,-0.16)$ \\
\hline
\end{tabular}

